\title{
Herkunftsbedingte Leistungsdisparitäten: Die Rolle von Selbstwirksamkeitsüberzeugungen und Unterstützungshandlungen von Eltern und Leistungserwartungen von Lehrpersonen
}

\section{Generalisierbarkeit eines Mediationsmodells für einsprachige und fremd- bzw. mehrsprachige Schülerinnen und Schüler}

\author{
Edith Niederbacher (D) Markus P. Neuenschwander \\ Online publiziert: 29. Juli 2020 \\ (C) Der/die Autor(en) 2020
}

Zusammenfassung Bei der Erklärung von herkunftsbedingten Leistungsdisparitäten spielen neben strukturellen Merkmalen familiäre und schulische Prozessmerkmale eine wichtige Rolle. Die vorliegende Studie versucht, den Zusammenhang zwischen dem elterlichen Ausbildungsniveau und Leistungen in Deutsch und Mathematik mittels Selbstwirksamkeitsüberzeugungen zur Lernunterstützung und Unterstützungshandlungen von Eltern sowie mittels Leistungserwartungen von Lehrpersonen zu erklären. Die Ergebnisse von Strukturgleichungsmodellen und Mediationsanalysen zeigten, dass der Zusammenhang zwischen dem elterlichen Ausbildungsniveau und den Leistungen in beiden Fächern durch Leistungserwartungen von Lehrpersonen sowie im Fach Deutsch durch elterliche Selbstwirksamkeitsüberzeugungen und Leistungserwartungen von Lehrpersonen (multiple Mediation) erklärt wird. Die Analysen wurden für zwei Gruppen (einsprachige und fremd- bzw. mehrsprachige Schülerinnen und Schüler) durchgeführt. Leistungsdisparitäten zwischen einsprachigen und fremd- bzw. mehrsprachigen Kindern wurden nicht durch unterschiedliche Zusammenhänge zwischen den Konzepten, sondern durch Mittelwertunterschiede in bestimmten Variablen erklärt.

Schlüsselwörter Leistungsdisparitäten · Selbstwirksamkeitsüberzeugungen · Unterstützungshandlungen · Lehrpersonenerwartungen · Familiensprache

\footnotetext{
E. Niederbacher $(\bowtie) \cdot$ Prof. Dr. M. P. Neuenschwander Zentrum Lernen und Sozialisation, Pädagogische Hochschule der Fachhochschule Nordwestschweiz, Obere Sternengasse 7, 4502 Solothurn, Schweiz

E-Mail: edith.niederbacher@fhnw.ch

Prof. Dr. M. P. Neuenschwander

E-Mail: markus.neuenschwander@fhnw.ch
} 


\title{
Disparities in academic achievement due to social background: The role of parents' self-efficacy and achievement-enhancing behavior and teachers' expectations of achievement
}

Generalizability of a mediation model for monolingual and foreign-language/ multilingual students

\begin{abstract}
Besides structural characteristics, process-based characteristics in family and school play an important role in explaining the effect of students' social background on their academic achievement. The aim of the present study is to explain the relationship between parents' educational attainment level and students' academic achievement in two domains (German and Mathematics) through parents' perceived self-efficacy in supporting their child's achievement, their achievement-enhancing behavior and through teachers' expectations of achievement. Results from structural equation models and mediation analysis indicate that the effect of parents' educational attainment level on students' achievement was explained by teachers' expectations of achievement in both domains and by parents' perceived self-efficacy and teachers' expectations of achievement (multiple mediation) in the domain German. The analyses were conducted for two groups (monolingual and foreign-language/ multilingual students). The pattern of relations was invariant across these groups of students. Disparities in academic achievement were explained by differences in their reported mean levels in some of the used variables.
\end{abstract}

Keywords Disparities in academic achievement - Language spoken at home · Teacher expectations · Parents' involvement · Perceived self-efficacy

\section{Einleitung}

Leistungen von Schülerinnen und Schülern hängen stark von strukturellen familiären Merkmalen, wie dem Ausbildungsniveau der Eltern, ab (Ehmke et al. 2004; Schneider und Pfost 2013). Mit dem Ausbildungsniveau der Eltern gehen gewisse schulbezogene Überzeugungen einher, welche über kulturelle Praxen (z. B. lernunterstützende Handlungen) die Lernprozesse und Leistungen der Kinder beeinflussen (Ehmke et al. 2006; Luplow und Smidt 2019). Diese Überzeugungen und Handlungen stellen Prozessmerkmale einer Familie dar, die den Zusammenhang zwischen dem elterlichen Ausbildungsniveau und den Leistungen von Schülerinnen und Schülern vermitteln (Watermann und Baumert 2006). Gemäß dem theoretischen Modell zur familiären Sozialisation von Eccles (1993) fungieren elterliche Selbstwirksamkeitsüberzeugungen zur Lernunterstützung (kurz ,Selbstwirksamkeitsüberzeugungen“) und Unterstützungshandlungen als relevante Prozessmerkmale, die den Zusammenhang zwischen elterlichem Ausbildungsniveau und schulischen Leistungen von Kindern erklären. Studien haben gezeigt, dass Eltern abhängig von ihrem Ausbildungsniveau unterschiedliche Selbstwirksamkeitsüberzeugungen und Unterstützungshandlungen entwickeln, welche die Leistungen ihrer Kinder beeinflussen (Hoover-Dempsey und Sandler 1997). 
Neben familiären Prozessmerkmalen wird der Zusammenhang zwischen Ausbildungsniveau und Leistungen der Kinder auch über schulische Prozessmerkmale erklärt. Das Modell zu Lehrpersonenüberzeugungen von Jussim et al. (1996) zeigt, dass Hintergrundmerkmale von Schülerinnen und Schülern die Leistungserwartungen von Lehrpersonen beeinflussen, welche wiederum Prädiktoren der Leistungen der Kinder sind. Studien belegen differenzielle Leistungserwartungen von Lehrpersonen in Abhängigkeit vom familiären Hintergrund der Kinder (Lorenz et al. 2016) und deren Bedeutung für die Leistungen (Wang et al. 2018).

Da die Akteurinnen und Akteure in Familie und Schule nicht ausschließlich gesondert, sondern auch im Zusammenspiel auf die Leistungen der Kinder einwirken, werden im vorliegenden Beitrag die genannten theoretischen Modelle verbunden. Im Rahmen der Kooperation zwischen Eltern und Lehrpersonen erhalten Lehrpersonen Informationen über das Ausbildungsniveau, die Selbstwirksamkeitsüberzeugungen und Unterstützungshandlungen der Eltern, welche ihre Leistungserwartungen sowie weiterführend die Leistungen der Kinder beeinflussen (Hoover-Dempsey et al. 1992; Neuenschwander 2014; Hofstetter 2017; Niederbacher und Neuenschwander 2017).

Mit dem vorliegenden Beitrag soll der Forschungsstand zur Entstehung von herkunftsbedingten Leistungsdisparitäten ergänzt werden, indem überprüft wird, ob der Zusammenhang zwischen dem Ausbildungsniveau von Eltern und den Leistungen von Schülerinnen und Schülern über Selbstwirksamkeitsüberzeugungen und Unterstützungshandlungen von Eltern, über Leistungserwartungen von Lehrpersonen oder gemeinsam über Eltern- und Lehrpersonenmerkmale vermittelt wird.

Ob herkunftsbedingte Leistungsdisparitäten durch Unterschiede in den Mittelwerten leistungsrelevanter Struktur- und Prozessmerkmale oder durch Unterschiede in den Zusammenhängen dieser Variablen erklärt werden, wurde bisher vor allem für verschiedene ethnische Gruppen geprüft (Shumow und Lomax 2002; Arens und Jude 2017). Studien zeigen jedoch, dass die Familiensprache neben der ethnischen Herkunft einen eigenen Erklärungsanteil an Leistungsdisparitäten hat (Konsortium PISA.ch 2010). Fremd- bzw. mehrsprachige ${ }^{1}$ Schülerinnen und Schüler erbringen schwächere Leistungen als einsprachige Kinder, deren Familiensprache der Unterrichtssprache entspricht (OECD 2016). Zwischen ein- und fremd- bzw. mehrsprachigen Familien bestehen Mittelwertunterschiede in ihren Selbstwirksamkeitsüberzeugungen und Unterstützungshandlungen (Hoover-Dempsey et al. 1992; Esser 2006). Ob sich die Zusammenhangsmuster zur Erklärung von Leistungsdisparitäten je nach Familiensprache der Kinder unterscheiden, wurde bislang kaum untersucht (Wong und Hughes 2006; Chudaske 2012). Daher wird in der zweiten Fragestellung überprüft, ob Leistungsdisparitäten von ein- und fremd- bzw. mehrsprachigen Schülerinnen und Schülern auf Mittelwertunterschiede in den verwendeten Variablen und/oder auf Unterschiede in den angenommenen direkten und indirekten Zusammenhängen zwischen dem Ausbildungsniveau der Eltern und den Leistungen der

\footnotetext{
${ }^{1}$ Als fremd- bzw. mehrsprachig gelten in vorliegendem Beitrag Schülerinnen und Schüler, die zuhause entweder eine Fremdsprache (z. B. Spanisch), mehrere Fremdsprachen (z. B. Spanisch und Portugiesisch) oder eine/mehrere Fremdsprachen sowie die Unterrichtssprache Deutsch (z. B. Spanisch und Deutsch) sprechen. Kinder, welche zuhause ausschließlich Deutsch sprechen und in der Schule eine weitere Fremdsprache erlernen, gelten in diesem Zusammenhang als einsprachig. Schweizerdeutsch wurde als Deutsch gezählt.
} 
Kinder zurückzuführen sind. Die Fragestellungen werden für die Fächer Deutsch und Mathematik am Beispiel einer Schweizer Stichprobe von Grundschülerinnen und -schülern geprüft.

\section{Theorie und Forschungsstand}

\subsection{Indirekte Zusammenhänge zwischen dem Ausbildungsniveau von Eltern und Leistungen von Schülerinnen und Schülern}

Das Ausbildungsniveau der Eltern erklärt schulische Kompetenzen von Kindern wesentlich (Ehmke et al. 2004; Sammons et al. 2008). Schülerinnen und Schüler in der Schweiz, deren Eltern nur die obligatorische Schulzeit absolviert und keinen höheren Abschluss erworben haben, erreichten im PISA-Test zur Lesekompetenz signifikant weniger Punkte (Coradi Vellacott und Wolter 2002). Der Effekt des elterlichen Ausbildungsniveaus auf Leistungen wird über familiäre und schulische Prozessmerkmale vermittelt (Jussim et al. 1996; Ehmke et al. 2006). Das Ausbildungsniveau der Eltern stellt einen Indikator für die kulturellen Ressourcen (Überzeugungen und Kompetenzen) einer Familie dar (Baumert und Maaz 2006). Es hat eine regulative Bedeutung für die kulturellen Praxen einer Familie (Watermann und Baumert 2006), welche sich in schulbezogenen Handlungen der Eltern äußern (Davis-Kean 2005; Luplow und Smidt 2019).

\subsubsection{Familiäre Prozessmerkmale: Elterliche Selbstwirksamkeitsüberzeugungen zur Lernunterstützung und Unterstützungshandlungen}

Gemäß dem Modell der familiären Sozialisation von Eccles (1993) beeinflusst das Ausbildungsniveau der Eltern ihre Überzeugungen, welche sich direkt sowie indirekt über ihre Handlungen auf die Leistungen der Kinder auswirken.

Eine Form von elterlichen Überzeugungen sind Selbstwirksamkeitsüberzeugungen, welche die Leistungen der Kinder beeinflussen (Bandura et al. 1996; HooverDempsey und Sandler 1997; Niederbacher und Neuenschwander 2017). Die Selbstwirksamkeit wird als eine domänen- und kontextspezifische Überzeugung einer Person verstanden, bestimmte Aufgaben erfolgreich bewältigen zu können (Zimmerman 1995; Bong und Skaalvik 2003). Elterliche Selbstwirksamkeitsüberzeugungen zur Lernunterstützung meinen demnach Überzeugungen von Eltern, ihrem Kind beim Lernen in einem Fach wirksam helfen zu können (Eccles und Harold 1993). Selbstwirksamkeitsüberzeugungen von Eltern sagen ihre schulbezogenen Handlungen vorher und sind leistungswirksam (Hoover-Dempsey und Sandler 1997; Tazouti und Jarlégan 2019). Hinsichtlich der Wirkung elterlicher Handlungen auf die Leistungen der Kinder ist der Forschungsstand aufgrund der Vielzahl an unterschiedlichen Operationalisierungen widersprüchlich (Fan und Chen 2001). Eine Form von Unterstützungshandlung ist die schulbezogene Kommunikation zwischen Eltern und Kindern, welche positiv mit schulischen Leistungen zusammenhängt (Jeynes 2005; Castro et al. 2015). Insgesamt zeigt sich, dass elterliche Überzeugungen stärker mit 
Leistungen zusammenhängen als elterliche Handlungen (Fan und Chen 2001; Wilder 2014). Basierend auf diesen Befunden lauten die Hypothesen:

H1 Der Zusammenhang zwischen dem elterlichen Ausbildungsniveau und den Leistungen der Schülerinnen und Schüler wird a) durch elterliche Selbstwirksamkeitsüberzeugungen, b) durch elterliche Unterstützungshandlungen und c) durch elterliche Selbstwirksamkeitsüberzeugungen und Unterstützungshandlungen (multiple Mediation) erklärt. H1d) Der Zusammenhang zwischen dem Ausbildungsniveau und den Leistungen verläuft stärker über die Selbstwirksamkeitsüberzeugungen als über die Unterstützungshandlungen der Eltern.

\subsubsection{Schulische Prozessmerkmale: Leistungserwartungen von Lehrpersonen}

Eine Form von Überzeugungen von Lehrpersonen stellen Erwartungen dar, die sich auf gegenwärtige und zukünftige Schülerinnen- und Schülerleistungen beziehen (Dusek und Joseph 1983). Das Modell von Jussim et al. (1996) zeigt, dass Leistungserwartungen von Lehrpersonen von Merkmalen der Schülerinnen und Schüler (z.B. frühere Leistungen, demografische Merkmale, Motivation) abhängen und die Leistungen beeinflussen.

Studien bestätigen den Effekt von Leistungserwartungen von Lehrpersonen auf die Leistungen der Schülerinnen und Schüler (Wang et al. 2018). Die Effekte von Leistungserwartungen auf Leistungen sind klein, aber stabil (Brophy 1983; Jussim und Harber 2005). Erwartungen von Lehrpersonen hängen nur zum Teil von den tatsächlichen Leistungen der Schülerinnen und Schüler ab (Südkamp et al. 2012). Sie sind von familiären Merkmalen der Kinder (z. B. ethnischer und sozialer Hintergrund; Jussim und Harber 2005; Lorenz et al. 2016) beeinflusst. Zudem hängen sie mit individuellen Merkmalen der Kinder wie Motivation (z. B. Anstrengungsvermeidung bzw. -bereitschaft; Lorenz et al. 2016) und Verhalten (z. B. Unterrichtsstörungen bzw. Regelkonformität; Timmermans et al. 2016) zusammen, weshalb solche Merkmale als Kontrollvariablen in Analysen einbezogen werden sollten. Studien zeigen, dass Erwartungen von Lehrpersonen systematisch verzerrt sind. Die Leistungserwartungen an Kinder aus ungünstigeren sozialen Verhältnissen sind niedriger, auch unter Kontrolle der Leistungen oder Anstrengungsbereitschaft der Kinder (Jussim et al. 1996; Stahl 2007; Lorenz et al. 2016). Die Erwartungen der Lehrpersonen beeinflussen ihre Interaktion mit den Schülerinnen und Schülern (Jussim et al. 2009), was deren Leistungen prägt (selbsterfüllende Prophezeiung; Lorenz 2018). Basierend darauf lautet die Hypothese:

H2 Der Zusammenhang zwischen dem elterlichen Ausbildungsniveau und den Leistungen der Schülerinnen und Schüler wird durch die Leistungserwartungen von Lehrpersonen erklärt.

\subsubsection{Das Zusammenspiel von elterlichen und schulischen Prozessmerkmalen}

Um das Zusammenspiel von elterlichen und schulischen Prozessmerkmalen hinsichtlich der Erklärung von herkunftsbedingten Leistungsdisparitäten zu prüfen, werden 
im vorliegenden Beitrag die genannten theoretischen Modelle (Eccles 1993; Jussim et al. 1996) verbunden. Wie dargelegt, hängen Leistungserwartungen von Lehrpersonen an die Schülerinnen und Schüler von deren familiären Herkunftsmerkmalen ab (Jussim et al. 1996; Wang et al. 2018). Über die Angaben im Schulanmeldungsformular zu Berufen der Eltern und in der Familie gesprochene(n) Sprache(n) schätzen Lehrpersonen das Ausbildungsniveau und den sprachlichen Hintergrund der Eltern ein, was ihre Leistungserwartungen an die Kinder beeinflusst (Brophy 1983). Weiter erhalten Lehrpersonen im Rahmen der Eltern-Lehrpersonen-Kooperation, beispielsweise in Elterngesprächen, Informationen zum Ausbildungsniveau und den Sprachkompetenzen der Eltern sowie über ihre schulrelevanten Überzeugungen und Handlungen (Hofstetter 2017). Die Kooperation zwischen Eltern und Lehrpersonen ist in der Schweiz per Gesetz verpflichtend (Schweizerisches Zivilgesetzbuch, Art. 302, Abs. 3, in Kraft seit 1978). Auf Grundlage kantonaler Bildungsgesetze findet mindestens einmal jährlich ein Elterngespräch statt, zudem werden weitere formale und informelle Kanäle für den Informationsaustausch genutzt (LCH 2017; Neuenschwander 2020). Studien zeigen, dass Leistungserwartungen von Lehrpersonen zusätzlich zum Einfluss der Herkunftsmerkmale - auch von den Selbstwirksamkeitsüberzeugungen und/oder den Unterstützungshandlungen der Eltern beeinflusst sind, auch nach Kontrolle von Schülermerkmalen (Hoover-Dempsey et al. 1992; Bakker et al. 2007; Kuperminc et al. 2008; Neuenschwander 2014; Hofstetter 2017). Wie Eltern- und Lehrpersonenmerkmale gemeinsam den Zusammenhang zwischen Ausbildungsniveau und Leistungen vermitteln, wurde bisher kaum untersucht. Daher wird folgende Hypothese geprüft:

H3 Der Zusammenhang zwischen dem elterlichen Ausbildungsniveau und den Leistungen der Schülerinnen und Schüler wird a) durch elterliche Selbstwirksamkeitsüberzeugungen und Leistungserwartungen von Lehrpersonen, b) durch elterliche Unterstützungshandlungen und Leistungserwartungen von Lehrpersonen sowie c) durch elterliche Selbstwirksamkeitsüberzeugungen, elterliche Unterstützungshandlungen und Leistungserwartungen von Lehrpersonen erklärt (multiple Mediationen).

\subsection{Fachunspezifische Zusammenhänge zwischen Struktur- und Prozessmerkmalen und Leistungen}

Wie das Ausbildungsniveau der Eltern, vermittelt über familiäre Prozessmerkmale, die Leistungen von Schülerinnen und Schülern beeinflusst, wurde in verschiedenen Fächern untersucht (Ehmke et al. 2006; Watermann und Baumert 2006). Die Höhe der Selbstwirksamkeitsüberzeugungen zur Lernunterstützung der Eltern und infolgedessen das Ausmaß ihrer Unterstützungshandlungen sind je nach Fach verschieden (Hoover-Dempsey und Sandler 1995). Die Zusammenhänge, wie elterliche Überzeugungen und Handlungen auf die Leistungen wirken, sind jedoch unabhängig vom Schulfach (Wilder 2014). Wie Lehrpersonenerwartungen die Leistungen beeinflussen (van den Bergh et al. 2010; Niederbacher und Neuenschwander 2017) sowie wie Lehrpersonenerwartungen den Einfluss von elterlichen Unterstützungs- 
handlungen auf Leistungen vermitteln, wurde ebenfalls in unterschiedlichen Fächern gezeigt (Bakker et al. 2007). Daher wird folgende Hypothese geprüft:

H4 Die postulierten indirekten Zusammenhänge zwischen Ausbildungsniveau und Leistungen über elterliche Selbstwirksamkeitsüberzeugungen, Unterstützungshandlungen und/oder Leistungserwartungen von Lehrpersonen finden sich in Deutsch und Mathematik in gleicher Weise.

\subsection{Leistungsdisparitäten zwischen einsprachigen und fremd- bzw. mehrsprachigen Schülerinnen und Schülern: Unterschiede in Mittelwerten und Zusammenhängen leistungsrelevanter Struktur- und Prozessmerkmale}

Die individuelle Fremd- bzw. Mehrsprachigkeit ist für das schulische Lernen relevant (Lengyel 2017). Schulische Leistungen sind an sprachliche Kompetenzen gebunden sind, weshalb gute Sprachkenntnisse in der Unterrichtssprache für den Bildungserfolg unabdingbar sind (Esser 2006). Studien zeigen, dass die in der Familie gesprochene Sprache - trotz enger Verknüpfung mit Migrationshintergrund, Ausbildungsniveau und sozioökonomischem Status (Chudaske 2012; Kempert et al. 2016; Kahn 2018) - einen eigenen Erklärungsanteil an Leistungen hat (Konsortium PISA.ch 2010; Stanat et al. 2010; Chudaske 2012; Gut et al. 2012; DittmannDomenichini 2014). Untersuchungen zu Leistungsdisparitäten zeigen, dass fremdbzw. mehrsprachige Kinder geringere Leistungen in sprachlichen und mathematischen Bereichen erbringen als einsprachige (OECD 2016; SKBF 2018; Hippmann et al. 2019). Werden verschiedene Sprachfacetten wie Lese-, Hör- und Schreibkompetenzen separat berücksichtigt, schneiden mehrsprachige Kinder in der Schulsprache Deutsch nicht generell schlechter ab, erbringen jedoch in gewissen Teilbereichen (Hörverstehen und pragmatisch-funktionales Schreiben) niedrigere Leistungen (Dittmann-Domenichini et al. 2011; Dittmann-Domenichini 2014).

Leistungsdisparitäten zwischen Gruppen werden über zwei Ansätze erklärt: über Mittelwertunterschiede in leistungsrelevanten Struktur- und Prozessmerkmalen und über unterschiedliche Zusammenhangsmuster zwischen den Variablen (Arens und Jude 2017). Hinsichtlich der Mittelwertunterschiede zwischen Eltern von ein- und fremd- bzw. mehrsprachigen Schülerinnen und Schülern wird in der Forschungsliteratur von Wechselwirkungen der Kategorien Ausbildungsniveau, Migrationshintergrund und Sprache berichtet. Mehr- bzw. fremdsprachige Familien zeichnen sich tendenziell durch ein niedrigeres Ausbildungsniveau sowie sozioökonomischen Status aus oder erleben in der Aufnahmegesellschaft eine Entwertung ihrer Bildungsabschlüsse (Ramm et al. 2004; Kristen und Granato 2007). Besitzen Eltern ein niedriges Ausbildungsniveau sowie unzureichende Sprachkenntnisse haben sie geringere Selbstwirksamkeitsüberzeugungen ihr Kind schulisch unterstützen zu können (Hoover-Dempsey et al. 1992; Bandura et al. 1996; Okagaki und Frensch 1998). Der Forschungsstand, ob sich Unterstützungshandlungen, operationalisiert über schulbezogene Kommunikation zwischen Eltern und Kind, je nach ethnischer oder sprachlicher Herkunft der Familien unterscheiden, ist uneinheitlich (kein Unterschied bzw. höheres/geringeres Ausmaß der Unterstützungshandlungen) (Wong und Hughes 2006). 
Lehrpersonen richten an fremd- bzw. mehrsprachige Kinder geringere Leistungserwartungen als an einsprachige Kinder, deren Familiensprache der Schulsprache entspricht (Stahl 2007; Carigiet Reinhard 2012).

Ob sich neben den Mittelwerten auch die Zusammenhangsmuster der Prozesse zur Erklärung von Leistungsdisparitäten zwischen ein- und fremd- bzw. mehrsprachigen Kindern unterscheiden, wurde bisher kaum erforscht (Wong und Hughes 2006; Chudaske 2012; Hippmann et al. 2019). Studien, die Leistungsdisparitäten zwischen Schülerinnen und Schülern basierend auf der ethnischen Herkunft oder dem Geburtsland untersuchen, berichten sowohl von Unterschieden in den Mittelwerten als auch in den Zusammenhängen der Variablen (LeFevre et al. 2010; van den Bergh et al. 2010; Arens und Jude 2017). Metastudien zeigen, dass die Zusammenhänge zwischen schulbezogenen Elternüberzeugungen bzw. -handlungen und Leistungen unabhängig von der Zugehörigkeit zu einer ethnischen Gruppe sind (Jeynes 2005). Basierend auf diesen Befunden werden folgende Hypothesen aufgestellt:

H5a (Mittelwertunterschiede): 1) Die Mittelwerte der Konstrukte Leistungen, elterliches Ausbildungsniveau, elterliche Selbstwirksamkeitsüberzeugungen und Leistungserwartungen von Lehrpersonen sind bei einsprachigen Schülerinnen und Schülern höher als bei fremd- bzw. mehrsprachigen Schülerinnen und Schülern (gerichtete Hypothese). 2) Der Mittelwert des Konstrukts elterliche Unterstützungshandlungen unterscheidet sich zwischen Eltern von ein- und fremd- bzw. mehrsprachigen Schülerinnen und Schülern (ungerichtete Hypothese).

H5b (Generalisierbarkeit des Mediationsmodells): Die postulierten direkten und indirekten Zusammenhänge sind für ein- und fremd- bzw. mehrsprachige Schülerinnen und Schüler gleich.

\section{Methode}

\subsection{Datengrundlage}

Die Datengrundlage stammt aus der Schweizer Längsschnittstudie „,Wirkungen der Selektion WiSel “2. Gearbeitet wurde mit Daten von Schülerinnen und Schülern aus den Kantonen Bern und Luzern, die zu zwei Messzeitpunkten im 5. und 6. Grundschuljahr (entspricht den letzten beiden Grundschuljahren der jeweiligen Kantone) in den Herbstsemestern 2011/12 ( $N=906)$ und 2012/13 $(N=778)$ schriftliche Fragebogen und Leistungstests bearbeiteten. Zwischen dem Einsatz von Fragebogen und Leistungstests lagen ca. 2-3 Wochen. Eltern und Klassenlehrpersonen der Kinder wurden in diesem Zeitraum ebenfalls schriftlich befragt.

Für die vorliegenden Analysen wurden diejenigen Schülerinnen und Schüler berücksichtigt, für welche die Angabe zur Familiensprache vorlag $(N=836)$ und welche entweder an beiden Messzeitpunkten (Längsschnitt LS, $N=690,82,5 \%$ ) oder

\footnotetext{
2 Wir danken dem Schweizerischen Nationalfonds für die Finanzierung der Studie (Projektnummer 100013_134594/1).
} 
nur zum ersten Messzeitpunkt (Querschnitt QS, $N=146,17,5 \%$ ) teilnahmen. Die LS-Gruppe hatte im Vergleich zur QS-Gruppe zum ersten Messzeitpunkt signifikant höhere Leistungen in Deutsch $\left(\mathrm{M}_{\mathrm{QS}}=-0,51, \mathrm{SD}_{\mathrm{QS}}=0,95, \mathrm{~N}_{\mathrm{QS}}=140 ; \mathrm{M}_{\mathrm{LS}}=-0,04\right.$, $\mathrm{SD}_{\mathrm{LS}}=1,01, \mathrm{~N}_{\mathrm{LS}}=673 ; \mathrm{t}(811)=5,06, p<0,001$, Cohen's $\left.d=0,5\right)$ und in Mathematik $\left(\mathrm{M}_{\mathrm{QS}}=-0,39, \mathrm{SD}_{\mathrm{QS}}=1,02, \mathrm{~N}_{\mathrm{QS}}=142 ; \mathrm{M}_{\mathrm{LS}}=-0,09, \mathrm{SD}_{\mathrm{LS}}=1,02, \mathrm{~N}_{\mathrm{LS}}=671\right.$; $\mathrm{t}(811)=3,15, p<0,01, d=0,3)$.

Die 836 Schülerinnen und Schüler (49,4\% weiblich, Alter: $M W=10,7$ Jahre, $S D=0,6, \operatorname{Min}=9, \operatorname{Max}=13$ ) stammten aus 54 Klassen in 22 Schulen. Davon waren 577 Kinder einsprachig mit deutscher Familiensprache $(69 \%)$ und 259 fremdbzw. mehrsprachig (31\%). 28,9\% besaßen eine ausländische Staatsbürgerschaft oder waren Doppelbürgerinnen bzw. -bürger. 92,6\% der Kinder waren in der Schweiz geboren.

Der Elternfragebogen wurde von 790 Eltern/Bezugspersonen der Schülerinnen und Schüler ausgefüllt (77,4\% Mütter, 11,4\% Väter, 5,6\% andere Bezugsperson, $5,6 \%$ keine Angabe). Es liegen für 94,5\% der Schülerinnen und Schüler Elternangaben vor.

Es füllten 52 der insgesamt 54 an der Studie teilnehmenden Klassenlehrpersonen (53,3\% weiblich, Alter: $M W=39,0$ Jahre, $S D=11,9$, Min $=22$, Max $=59$, Unterrichtserfahrung: $M W=15,6$ Jahre, $S D=10,9$, Min $=0$, Max $=40$ ) in Bezug auf jede Schülerin bzw. jeden Schüler Fragebogen aus $(N=813)$. Somit liegen für 97,2\% der Schülerinnen und Schüler Daten aus Lehrpersonenperspektive vor.

\subsection{Messinstrumente und Operationalisierung}

Im Folgenden werden die in den Analysen verwendeten Konzepte vorgestellt (Deskriptive Angaben in Tab. 1).

Die Leistungen der Schülerinnen und Schüler in Deutsch und Mathematik wurden über standardisierte, lehrplanvalide Leistungstests (Moser et al. 2011) erhoben. Die Deutschtests für das 5. und 6. Grundschuljahr beinhalteten Aufgaben zu den Kompetenzbereichen Wortschatz, Grammatik, Orthografie und Textverständnis $\left(\mathrm{t}_{1}\right.$ : 6 Aufgaben mit 83 Items, $\mathrm{t}_{2}$ : 10 Aufgaben mit 85 Items). Die Mathematiktests beinhalteten Aufgaben zu den Bereichen Grundoperationen, Zahlenfolgen, Proportionen, Sachrechnen sowie Brüche und Dezimalzahlen ( $t_{1}: 7$ Aufgaben mit 39 Items, $\mathrm{t}_{2}$ : 10 Aufgaben mit 45 Items). Split-half-Reliabilitäten erste vs. zweite Testhälfte (D: $\mathrm{t}_{1}: r_{S H}=0,80, \mathrm{t}_{2}: r_{S H}=0,82, \mathrm{M}: \mathrm{t}_{1}: r_{S H}=0,80, \mathrm{t}_{2}: r_{S H}=0,87$ ) sowie gerade vs. ungerade Items (D: $\left.\mathrm{t}_{1}: r_{S H}=0,82, \mathrm{t}_{2}: r_{S H}=0,95, \mathrm{M}: \mathrm{t}_{1}: r_{S H}=0,82, \mathrm{t}_{2}: r_{S H}=0,91\right)$ der Leistungstests waren in beiden Fächern zufriedenstellend. Eine Teilmenge der Items des ersten Tests wurde zum zweiten Messzeitpunkt wiederholt vorgelegt (Ankeritem-Design). Auf Grundlage der Item-Response-Theorie (Yen und Fitzpatrick 2006) wurde mit der Software R die Linking-Methode von Haberman (2009) angewendet, wodurch die Leistungen der zwei Messzeitpunkte auf einer Metrik abgebildet sind. Als Kompetenzwerte wurden Weighted-Likelihood-Werte (Warm 1989) ermittelt.

Im Fragebogen für Schülerinnen und Schüler gaben die Kinder zur Erfassung der Familiensprache alle in der Familie gesprochen Sprachen an. Es wurde eine binäre Variable ((1) ,einsprachig Deutsch“ und (0) „fremd- bzw. mehrsprachig“) gebildet. Die eigene Anstrengungsvermeidung schätzten die Kinder anhand von drei Items 


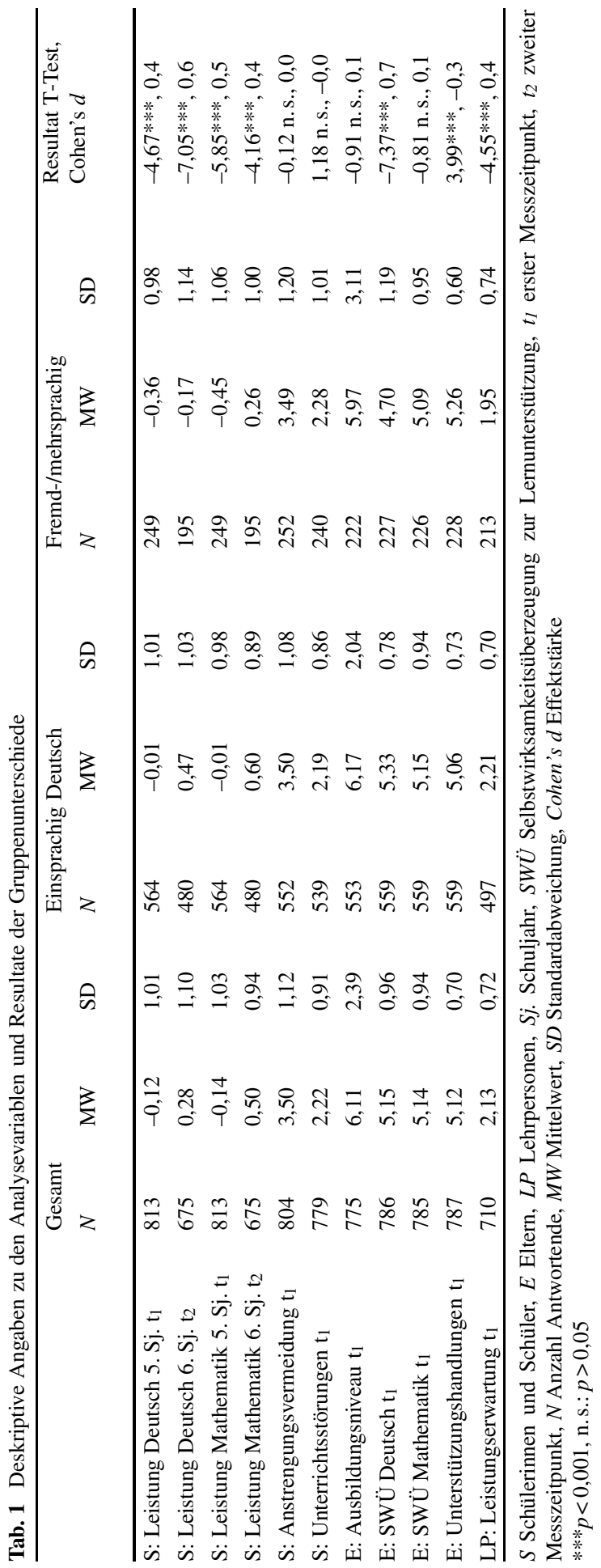


(Beispielitem: „Ich mache lieber Aufgaben, die ich bereits kenne, als solche, bei denen ich neu lernen muss, wie man sie löst.“, eigene Übersetzung nach Midgley et al. 2000) auf einer sechsstufigen Skala ((1) ,stimmt überhaupt nicht“ bis (6) „stimmt voll und ganz") ein (Cronbach's $\alpha=0,63$ ). Die Unterrichtsstörungen der Schülerinnen und Schüler wurden über drei Items (Beispielitem: „Bei Gruppenarbeiten rede ich meistens über andere Dinge als wir sollten.“, Neuenschwander et al. 2003) auf einer sechsstufigen Skala ((1) ,stimmt überhaupt nicht“ bis (6) ,stimmt voll und ganz") erfasst $(\alpha=0,74)$.

Das Ausbildungsniveau der ersten Bezugsperson wurde über die Frage „Welches ist die höchste Ausbildung, welche Sie abgeschlossen haben?“ erfasst (elfstufige Skala: (1) „Primarschule (bis 7 Schuljahre)“, (2) „Obligatorische Schule (8 oder 9 Schuljahre)“, (3) „10. Schuljahr, Au-pair-Jahr, Vorlehre, Haushaltsjahr“, (4) „Anlehre“, (5) „Lehre oder Vollzeitberufsschule“, (6) „Diplommittelschule, Fachmittelschule, Verkehrsschule usw.“, (7) „Gymnasium, Kantonsschule, Lehrpersonen-Seminar, Berufsmaturitätsschule“, (8) „Höhere Fach- oder Berufsausbildung“, (9) „Höhere Fachschule (HTL, HWV, HFG, HFS usw.)“, (10) „Fachhochschule oder Pädagogische Hochschule“, (11) „Universität oder ETH“). Zur Angabe der Selbstwirksamkeitsüberzeugungen zur Lernunterstützung beantwortete die Bezugsperson zwei fachspezifische Fragen „Wie gut können Sie Ihr Kind beim Lernen in folgenden Fächern unterstützen?" für die Fächer Deutsch und Mathematik auf einer sechsstufigen Skala ((1), ,überhaupt nicht gut“ bis (6) ,sehr gut“, in Anlehnung an HooverDempsey und Sandler 2005). Die Unterstützungshandlungen beziehen sich auf die Facette der schulbezogenen Kommunikation mit dem Kind und wurden über drei Items (Beispielitem: „Ich spreche häufig mit meinem Kind über Dinge, die es in der Schule gelernt hat.", in Anlehnung an Cheung und Pomerantz 2011; Neuenschwander et al. 2013) mit einer sechsstufigen Skala ((1) „stimmt überhaupt nicht“ bis (6) ,stimmt voll und ganz“) erfasst $(\alpha=0,64)$.

Die Leistungserwartungen der Lehrpersonen an jede Schülerin/jeden Schüler ihrer Klasse wurden mit einer fächerübergreifenden Frage „Was für eine Leistung erbringt diese Schülerin bzw. dieser Schüler in den drei Hauptfächern (Deutsch, Französisch und Mathematik) im Vergleich zu den anderen Kindern Ihrer Klasse?" (Eigenkonstruktion; Neuenschwander et al. 2012) auf einer dreistufigen Skala ((1) „unterdurchschnittliche“, (2) ,durchschnittliche“ und (3) ,überdurchschnittliche") erhoben.

Die abhängige Variable (Deutsch- bzw. Mathematikleistung im 6. Grundschuljahr) in den Modellen stammt vom zweiten Messzeitpunkt, die übrigen Variablen vom ersten Messzeitpunkt. Die Konstrukte Leistung im 5. Schuljahr, Anstrengungsvermeidung und Unterrichtsstörungen fungieren in den vorliegenden Analysen als Kontrollvariablen.

\subsection{Auswertungsverfahren}

Die postulierten Mittelwertunterschiede zwischen ein- und mehr- bzw. fremdsprachigen Schülerinnen und Schülern (H5a) wurden mittels T-Tests in SPSS Version 25 geprüft. Es wurden die Effektstärken der Gruppenunterschiede berechnet (Cohen's $d$; 
Werte ab 0,2 entsprechen einem kleinen, ab 0,5 einem mittleren und ab 0,8 einem großen Effekt; Cohen 1988).

Um die Generalisierbarkeit der Zusammenhangsmuster für beide Gruppen (H5b) zu prüfen, wurden Multigroup-Strukturgleichungsmodelle mit Mplus Version 8 berechnet (Muthén und Muthén 1998-2017). Die hierarchische Datenstruktur (Schülerinnen und Schüler in Klassen) wurde mithilfe des Analysetyps „type = complex“ kontrolliert. Die Analysen wurden mittels dem gegenüber der Verletzung der Normalverteilungsannahme robustem Standardfehlerschätzer MLR durchgeführt (Muthén und Satorra 1995). Zum Umgang mit fehlenden Werten (durchschnittlich 7,8\%) wurde FIML (Full Information Maximum Likelihood-Estimation) verwendet, welches auch beim Vorliegen systematisch fehlender Werte empfohlen wird (Graham 2009). Zur Bewertung der Strukturgleichungsmodelle wurden die ChiQuadrat-Werte $\left(\chi^{2}\right)$ und Freiheitsgrade $(d f)$ sowie folgende Fit-Indizes berichtet: Comparative Fit Index (CFI, ab 0,95), Root Mean Square Error of Approximation (RMSEA, unter 0,06), Standardized Root Mean Square Residual (SRMR, unter 0,08) (Hu und Bentler 1999), Gamma hat ( $\gamma^{\gamma}$, ab 0,95; Hu und Bentler 1998; Marsh et al. 2004) und McDonald's (1989) Noncentrality Index (Mc Donald's NCI (Mc), ab 0,90; McDonald und Marsh 1990). Die Werte für $\wedge^{\wedge}$ und Mc wurden basierend auf den Formeln in Hu und Bentler (1998) berechnet. Zur Testung der Modelle auf Gruppenunterschiede wurden Differenzwerte für die Modellfit-Indizes CFI, $\wedge \gamma$ und Mc berechnet, da diese im Gegensatz zum $\chi^{2}$-Differenztest unabhängig von der Modellkomplexität und der Stichprobengröße sind (Cheung und Rensvold 2002). Als Cutoff-Werte gelten die Werte $-0,01$ für $\Delta$ CFI, $-0,001$ für $\Delta^{\wedge} \gamma$ und $-0,02$ für $\Delta$ Mc (Cheung und Rensvold 2002).

Die postulierten indirekten Zusammenhänge zwischen den Variablen (H1a-c, H2, $\mathrm{H} 3 \mathrm{a}-\mathrm{c}$ ) wurden mittels Mediationsanalysen über die Funktion „Indirect“ in Mplus berechnet. Der Zusammenhang zwischen zwei Variablen wird vollständig durch eine oder mehrere Mediatorvariablen vermittelt, wenn der indirekte Effekt signifikant und der direkte Effekt nicht signifikant ist (Zhao et al. 2010). Zur Beantwortung der Mediationshypothesen wurden die Koeffizienten, Standardfehler und 95\%-Konfidenzintervalle der spezifischen indirekten sowie direkten Effekte berichtet (Zhao et al. 2010; Loeys et al. 2015). Der Vergleich der Stärke zweier Mediationen (H1d) wurde mithilfe der Funktion „Model Constraint“ durch die Testung der Differenzwerte der Koeffizienten der indirekten Pfade auf Signifikanz berechnet (Lau und Cheung 2012).

\section{Ergebnisse}

Zur Prüfung von Hypothese 5a wurden für die verwendeten Variablen Mittelwertunterschiede zwischen ein- und fremd- bzw. mehrsprachigen Schülerinnen und Schülern sowie deren Eltern und Lehrpersonen berechnet (Tab. 1). Fremd- bzw. mehrsprachige Schülerinnen und Schüler erbringen zu beiden Messzeitpunkten in Deutsch und Mathematik signifikant niedrigere Leistungen als einsprachige. Einsprachige und fremd- bzw. mehrsprachige Eltern unterscheiden sich nicht signifikant in ihrem Ausbildungsniveau. Die Selbstwirksamkeitsüberzeugung im Fach Deutsch ist 


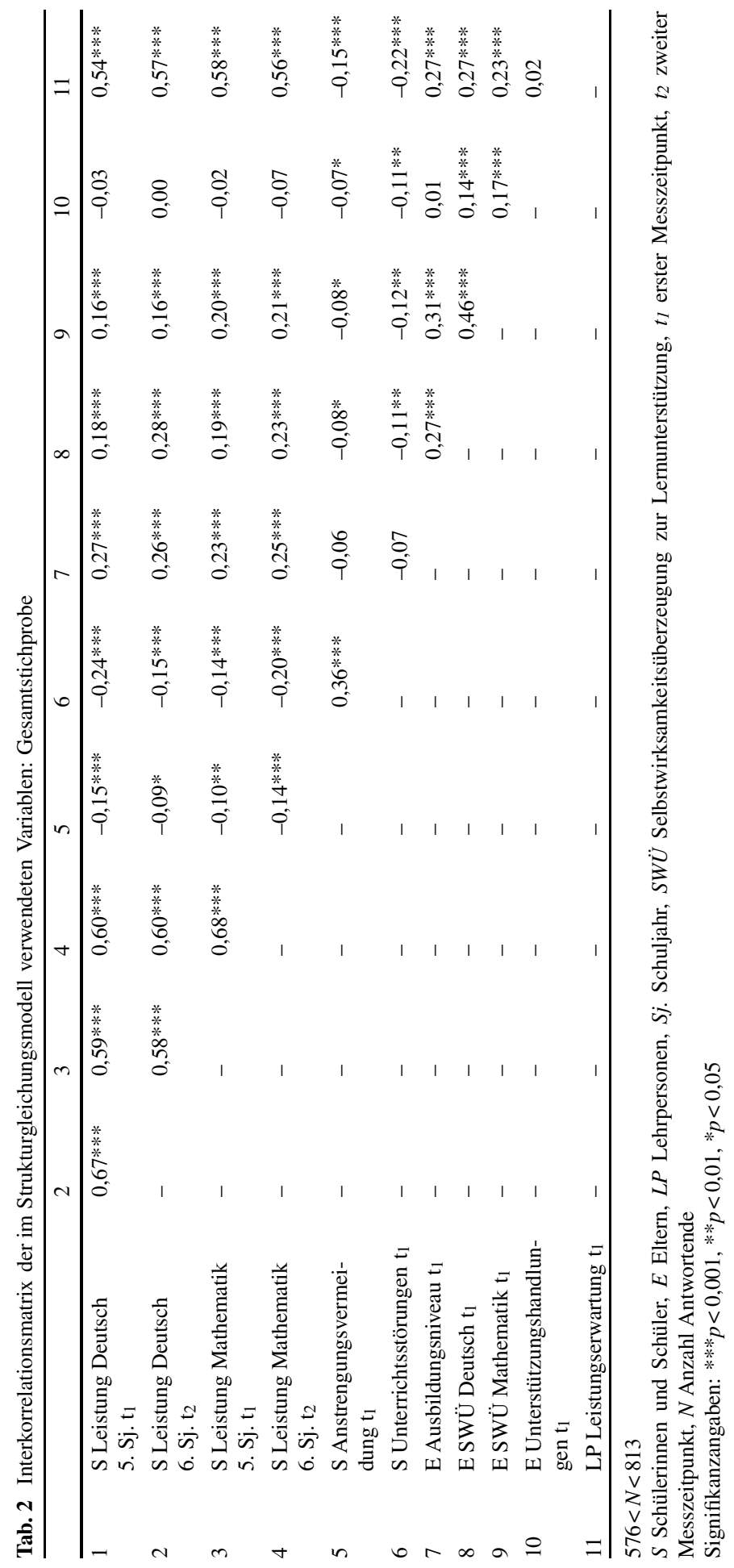




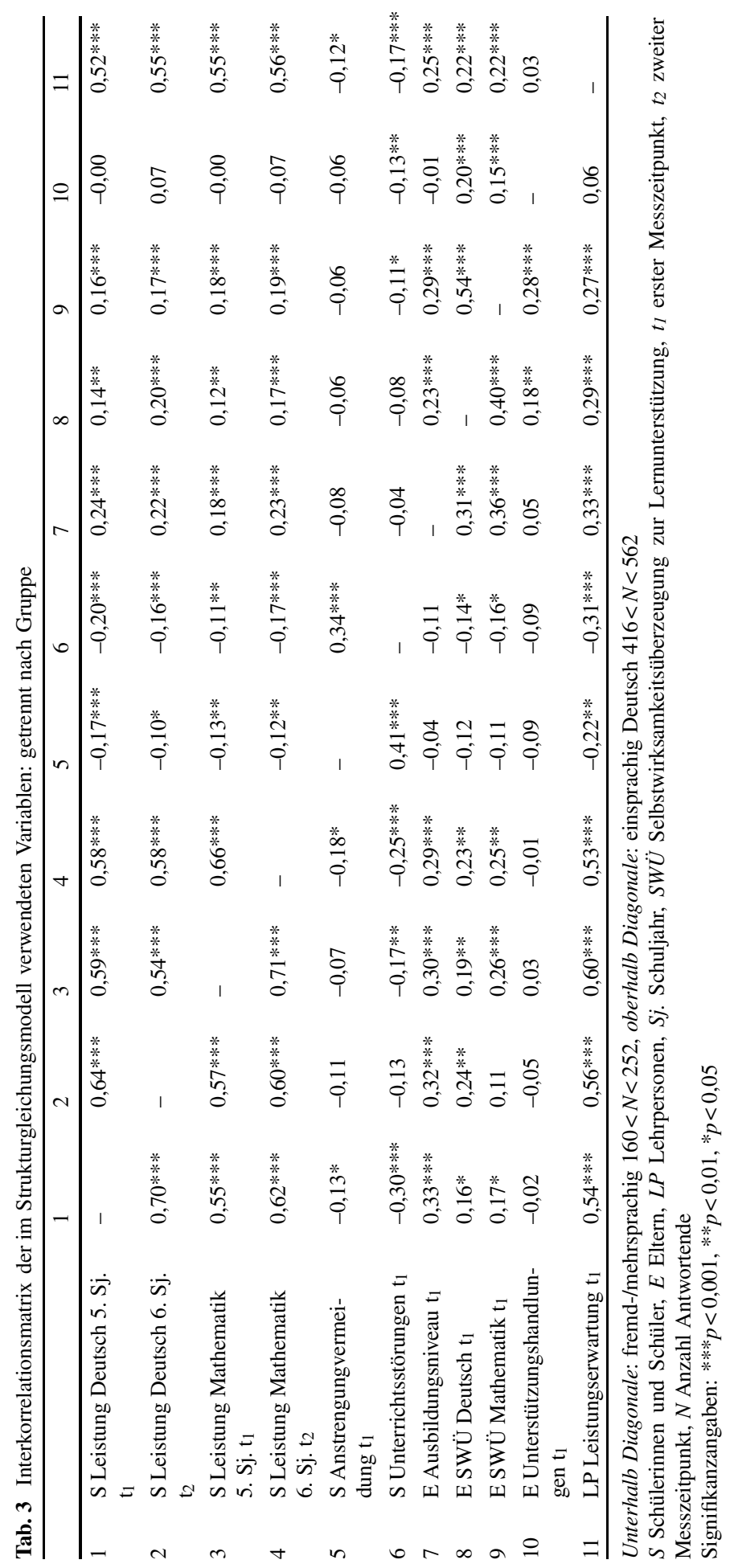


bei einsprachigen Eltern höher als bei fremd- bzw. mehrsprachigen Eltern. Hinsichtlich der Selbstwirksamkeitsüberzeugung in Mathematik unterscheiden sie sich nicht. Die Lehrpersonen haben höhere Leistungserwartungen an einsprachige Kinder als an fremd- bzw. mehrsprachige (H5a1 teilweise bestätigt). Fremd- bzw. mehrsprachige Eltern berichten ein höheres Ausmaß an Unterstützungshandlungen als einsprachige Eltern (H5a2 bestätigt).

Zur Prüfung der Zusammenhänge zwischen den Variablen wurden zunächst Korrelationen mit SPSS berechnet (Tab. 2). Das Ausbildungsniveau korreliert positiv mit den elterlichen Selbstwirksamkeitsüberzeugungen in Deutsch und Mathematik, den Leistungserwartungen der Lehrpersonen und den Leistungen in beiden Fächern zu beiden Messzeitpunkten. Die Unterstützungshandlungen der Eltern hängen positiv mit ihren Selbstwirksamkeitsüberzeugungen zusammen, nicht aber mit ihrem Ausbildungsniveau. Die Leistungserwartungen der Lehrpersonen hängen neben den Leistungen der Kinder mit dem Ausbildungsniveau und den Selbstwirksamkeitsüberzeugungen der Eltern zusammen, nicht aber mit deren Unterstützungshandlungen. Es zeigt sich ein signifikanter negativer Zusammenhang zwischen den Leistungserwartungen der Lehrpersonen und den Kontrollvariablen Anstrengungsvermeidung und Unterrichtsstörungen der Schülerinnen und Schüler.

Der Zusammenhang zwischen den Unterstützungshandlungen und der Selbstwirksamkeitsüberzeugung in Mathematik ist bei fremd- bzw. mehrsprachigen Eltern stärker als bei einsprachigen (Fisher's $Z=1,73, p<0,05, d=0,1$ ). Bei den übrigen Korrelationen gibt es keine signifikanten Gruppenunterschiede (Tab. 3).

Zur Testung der Hypothese 5b wurde geprüft, ob die postulierten Zusammenhänge zwischen den Variablen abhängig von der Familiensprache der Schülerinnen und Schüler variierten. Dafür wurden zuerst die drei latenten Konstrukte Unterstützungshandlungen, Anstrengungsvermeidung und Unterrichtsstörungen auf Messinvarianz zwischen den Gruppen überprüft. Jedes Messmodell wurde mit je drei Items und einem entsprechenden Fehlerterm pro Item spezifiziert. Jedes Item lud nur auf eine latente Variable. Die latenten Konstrukte wurden miteinander korreliert. Der Modellfit wurde für die drei Messmodelle gemeinsam berechnet $\left(N=835, \chi^{2}=49,67, d f=24\right.$, Scaling Correction Factor 0,99, CFI $=0,98$, RMSEA $=0,04$, SRMR $=0,03$ ). Beim Konstrukt Anstrengungsvermeidung wurde gemäß „Modification Indices“ eine Fehlerkorrelation zwischen zwei Items zugelassen, welche aufgrund eines ähnlichen Itemlauts inhaltlich begründbar war und den Modellfit der drei Konstrukte signifikant verbesserte $\left(N=835, \chi^{2}=20,22\right.$, $d f=23$, Scaling Correction Factor $1,00, \mathrm{CFI}=1,00, \mathrm{RMSEA}=0,00, \mathrm{SRMR}=0,02$; $\left.\Delta \chi^{2}=38,30, d f=1, p<0,001\right)$. Die drei Messmodelle wiesen auch im Multigroupverfahren einen guten Fit auf ( $N=835$, davon $\mathrm{N}_{\text {Einsprachige }}=576, \mathrm{~N}_{\text {Fremd-Mehrsprachige }}=259$; $\chi^{2}=50,23, d f=46, \mathrm{CFI}=1,00$, RMSEA $=0,02, \mathrm{SRMR}=0,03, \wedge \gamma=1,00, \mathrm{Mc}=1,00$ ). Das Modell mit gleichgesetzten Faktorladungen $\left(\chi^{2}=54,60, d f=52, \mathrm{CFI}=1,00\right.$, RMSEA $=0,01, \operatorname{SRMR}=0,03, \wedge \gamma=1,00, \mathrm{Mc}=1,00)$ unterschied sich nicht signifikant vom Modell ohne Restriktionen zwischen den Gruppen $(\Delta \mathrm{CFI}=-0,00$, $\left.\Delta^{\wedge} \gamma=-0,00, \Delta \mathrm{Mc}=-0,00\right)$. Demnach lag metrische Messinvarianz für die drei latenten Konstrukte vor, wodurch die Voraussetzung für einen Gruppenvergleich bei den Strukturgleichungsmodellen gegeben war (Christ und Schlüter 2012). 
Auf Basis der theoretischen Modelle wurde in den Strukturgleichungsmodellen (Abb. 1 und 2) der Zusammenhang zwischen dem Ausbildungsniveau der Eltern (X) und den Leistungen der Schülerinnen und Schüler in Deutsch und Mathematik (Y) über drei Mediatorvariablen modelliert: elterliche Selbstwirksamkeitsüberzeugungen in Deutsch bzw. Mathematik $\left(\mathrm{M}_{1}\right)$, elterliche Unterstützungshandlungen $\left(\mathrm{M}_{2}\right)$ und Leistungserwartungen von Lehrpersonen $\left(\mathrm{M}_{3}\right)$. Es wurden sieben indirekte Pfade postuliert (H1a-c, H2, H3a-c; Tab. 4). Als Kontrollvariablen wurden als Leistungsmaß die Vorleistung der Kinder im 5. Schuljahr $\left(\mathrm{C}_{1}\right)$, als Motivationsmaß die Anstrengungsvermeidung $\left(\mathrm{C}_{2}\right)$ und als Verhaltensmaß die Unterrichtsstörungen $\left(\mathrm{C}_{3}\right)$ der Kinder als Prädiktoren der Leistungserwartungen der Lehrperson einbezogen. Zur Replikation der Befunde wurden die Analysen für Deutsch und Mathematik getrennt vorgenommen (H4). In einem ersten Schritt wurden die Zusammenhänge in den Strukturgleichungsmodellen der beiden Fächer Deutsch (D) und Mathematik (M) auf Unterschiede zwischen den Gruppen überprüft.

Die Multigroup-Strukturgleichungsmodelle ohne Restriktion zwischen den Gruppen $\left(N=775\right.$, davon $\left.\mathrm{N}_{\text {Einsprachige }}=553, \mathrm{~N}_{\text {Fremd-/Mehrsprachige }}=222\right)$ weisen für beide Fächer gute Modellfitwerte auf $\left(\mathrm{D}: \chi^{2}=209,46, d f=140, \mathrm{CFI}=0,96, \mathrm{RMSEA}=0,04\right.$, SRMR $=0,06, \quad \wedge \gamma=0,99, \quad \mathrm{Mc}=0,96 ; \quad \mathrm{M}: \chi^{2}=231,59, \quad d f=140, \quad \mathrm{CFI}=0,95$, $\mathrm{RMSEA}=0,04, \mathrm{SRMR}=0,06, \wedge \gamma=0,98, \mathrm{Mc}=0,94)$. Die Modelle mit gleichgesetzten Pfadkoeffizienten zwischen den Gruppen (D: $\chi^{2}=228,08, \quad d f=157$, $\mathrm{CFI}=0,96, \mathrm{RMSEA}=0,03, \mathrm{SRMR}=0,06, \wedge \gamma=0,99, \mathrm{Mc}=0,96 ; \mathrm{M}: \chi^{2}=254,32$, $d f=157, \mathrm{CFI}=0,95, \mathrm{RMSEA}=0,04, \mathrm{SRMR}=0,06, \wedge \gamma=0,98, \mathrm{Mc}=0,94)$ sind in beiden Fächern nicht signifikant verschieden von den Modellen mit frei geschätzten Koeffizienten (D: $\Delta \mathrm{CFI}=-0,00, \Delta^{\wedge} \gamma=-0,00, \Delta \mathrm{Mc}=-0,00 ; \mathrm{M}: \Delta \mathrm{CFI}=-0,00$, $\left.\Delta^{\wedge} \gamma=-0,00, \Delta \mathrm{Mc}=-0,00\right)$. Demnach unterscheiden sich die Zusammenhänge zwischen den Variablen in den Fächern Deutsch und Mathematik nicht in Abhängigkeit von der Familiensprache der Schülerinnen und Schüler (H5b bestätigt). Aus diesem Grund werden im Folgenden nicht die Ergebnisse der Strukturgleichungsmodelle im Multigroup-Verfahren, sondern die Ergebnisse der Modelle mit der gesamten Stichprobe dargestellt.

Das Strukturgleichungsmodell zur Erklärung des Zusammenhangs zwischen dem Ausbildungsniveau der Eltern und der Leistung im 6. Schuljahr im Fach Deutsch (Abb. 1) weist einen guten Modellfit auf $\left(N=775, \chi^{2}=136,97, d f=64, \mathrm{CFI}=0,96\right.$, RMSEA =0,04, SRMR=0,05). Es zeigt sich ein signifikanter positiver Zusammenhang zwischen dem Ausbildungsniveau der Eltern und der elterlichen Selbstwirksamkeitsüberzeugung im Fach Deutsch. Zwischen dem Ausbildungsniveau und den Unterstützungshandlungen der Eltern gibt es keinen Zusammenhang. Die elterliche Selbstwirksamkeitsüberzeugung in Deutsch hängt mit den elterlichen Unterstützungshandlungen sowie mit den Leistungserwartungen der Lehrpersonen zusammen, auch unter Kontrolle der Effekte der Vorleistung, Anstrengungsvermeidung und Unterrichtsstörungen der Kinder auf die Leistungserwartungen der Lehrpersonen. Die elterliche Selbstwirksamkeitsüberzeugung in Deutsch korrespondiert mit der Deutschleistung der Kinder im 6. Schuljahr. Die Unterstützungshandlungen haben keinen signifikanten Zusammenhang mit der Leistung der Kinder und den Leistungserwartungen der Lehrpersonen. Die Leistungserwartungen hängen vom Ausbildungsniveau der Eltern ab und korrelieren mit der Deutschleistung der Schülerinnen 


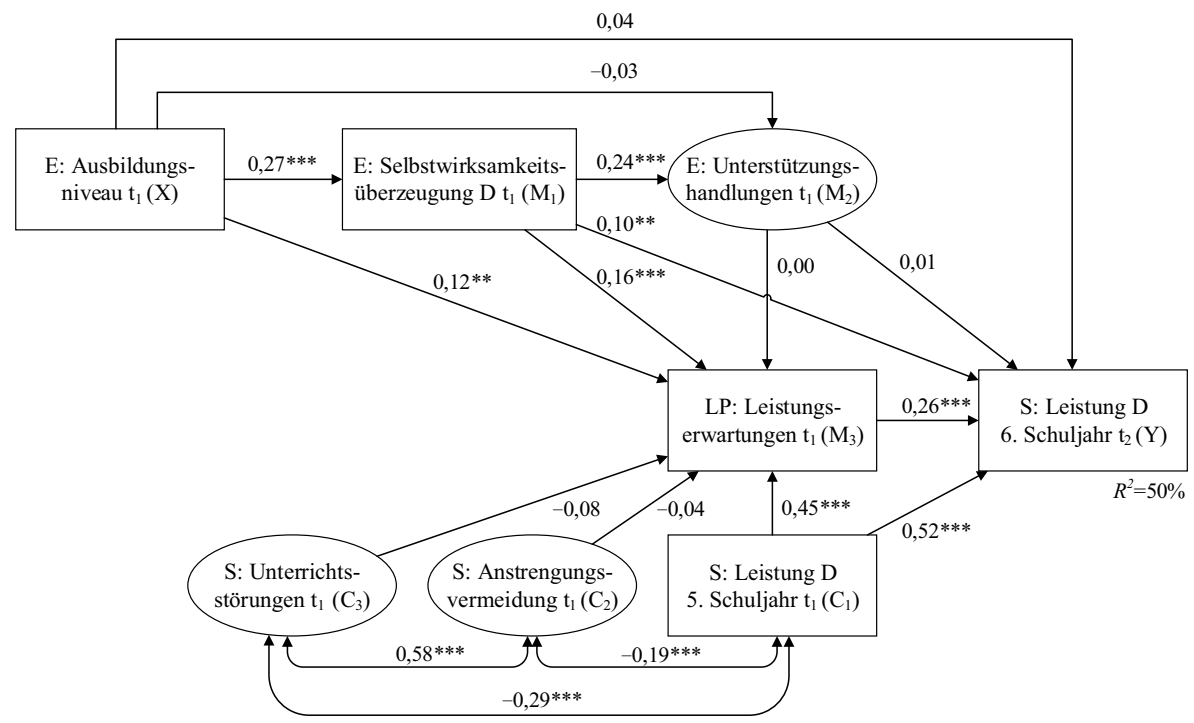

Abb. 1 Strukturgleichungsmodell zur Erklärung des Zusammenhangs zwischen dem elterlichen Ausbildungsniveau und den Leistungen von Schülerinnen und Schülern im Fach Deutsch (standardisierte $\beta$-Koeffizienten der direkten Pfade und Signifikanzen), $N=775$. ( $S$ Schülerinnen und Schüler, $E$ Eltern, $L P$ Lehrpersonen, $D$ Deutsch, $t_{l}$ erster Messzeitpunkt, $t_{2}$ zweiter Messzeitpunkt, $N$ Stichprobengröße, $X$ Unabhängige Variable, $M_{1} / M_{2} / M_{3}$ Mediatorvariablen, $Y$ Abhängige Variable, $C_{1} / C_{2} / C_{3}$ Kontrollvariablen, $R^{2}$ Varianzaufklärung. Signifikanzangaben: $* * * p<0,001, * * p<0,01, * p<0,05)$

und Schüler. Die Kontrollvariable Leistung im Fach Deutsch im 5. Schuljahr hängt stark mit der Leistung im 6. Schuljahr sowie den Leistungserwartungen der Lehrperson zusammen. Zwischen Anstrengungsvermeidung respektive Unterrichtsstörungen der Kinder und den Leistungserwartungen der Lehrperson gibt es keine Zusammenhänge. Die aufgeklärte Varianz der Leistungen im Fach Deutsch im 6. Schuljahr beträgt $50 \%$.

Die Ergebnisse der Mediationsanalysen (Tab. 4) zeigen, dass der Zusammenhang zwischen dem elterlichen Ausbildungsniveau und der Deutschleistung im 6. Schuljahr über die Selbstwirksamkeitsüberzeugung der Eltern $\left(\mathrm{X} \rightarrow \mathrm{M}_{1} \rightarrow \mathrm{Y}: \beta=0,03\right.$, $S E \beta=0,01, p<0,01, C I \beta[0,01 ; 0,04]$, H1a bestätigt) und über die Leistungserwartungen der Lehrpersonen $\left(\mathrm{X} \rightarrow \mathrm{M}_{3} \rightarrow \mathrm{Y}: \beta=0,03, S E \beta=0,01, p<0,05, C I \beta\right.$ $[0,01 ; 0,06], \mathrm{H} 2$ bestätigt) erklärt wird. Hinsichtlich des gemeinsamen Wirkens von Eltern und Lehrpersonen zeigt sich, dass der Zusammenhang des Ausbildungsniveaus und der Deutschleistung über die elterliche Selbstwirksamkeitsüberzeugung und die Leistungserwartungen der Lehrpersonen vermittelt wird $\left(\mathrm{X} \rightarrow \mathrm{M}_{1} \rightarrow \mathrm{M}_{3} \rightarrow \mathrm{Y}\right.$ : $\beta=0,01, S E \beta=0,01, p<0,01, C I \beta[0,00 ; 0,02], \mathrm{H} 3$ a bestätigt). Da die elterlichen Unterstützungshandlungen nicht mit den Leistungserwartungen der Lehrperson und den Leistungen der Kinder zusammenhängen, gibt es keine weiteren signifikanten Mediationseffekte (H1b, 1c, 3b und 3c nicht bestätigt). Die Hypothese 1d, in welcher angenommen wurde, dass der Zusammenhang zwischen dem Ausbildungsniveau und den Leistungen stärker über die Selbstwirksamkeitsüberzeugungen als über Unterstützungshandlungen wirkt, wird im Fach Deutsch bestätigt (Differenzwert 


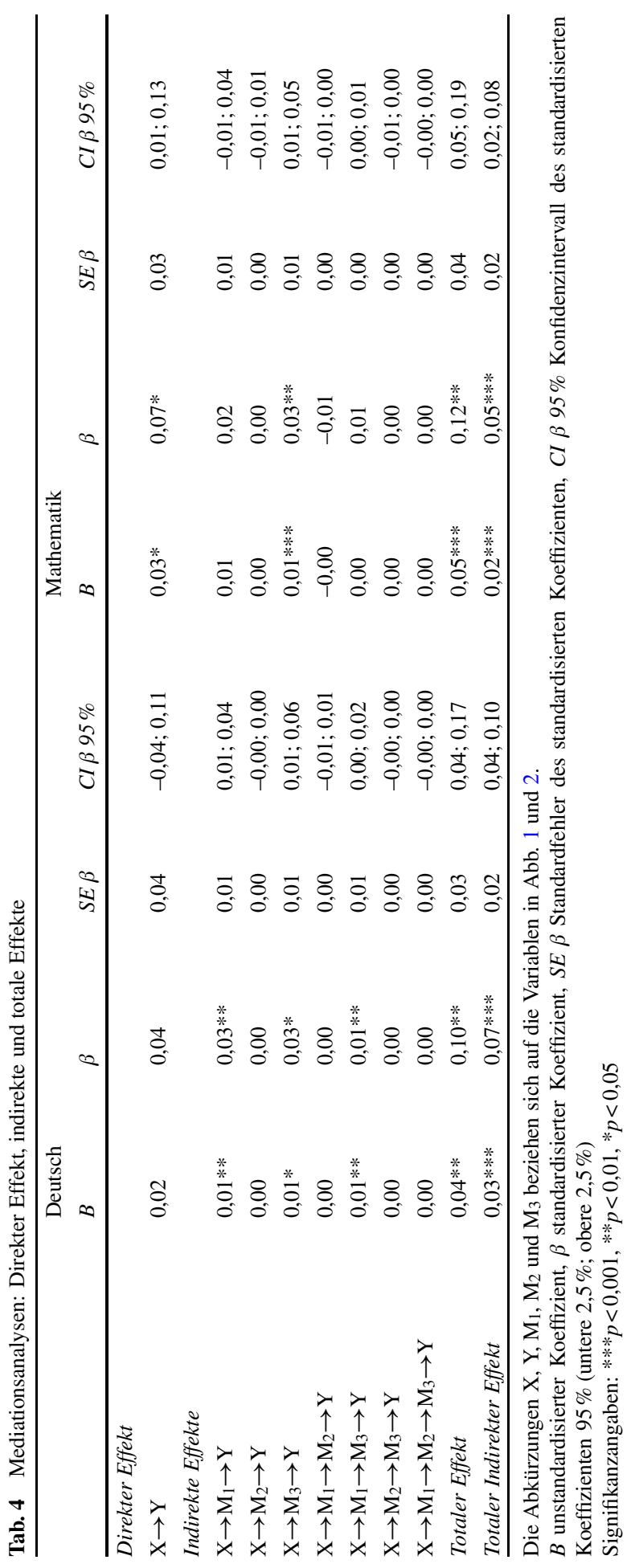




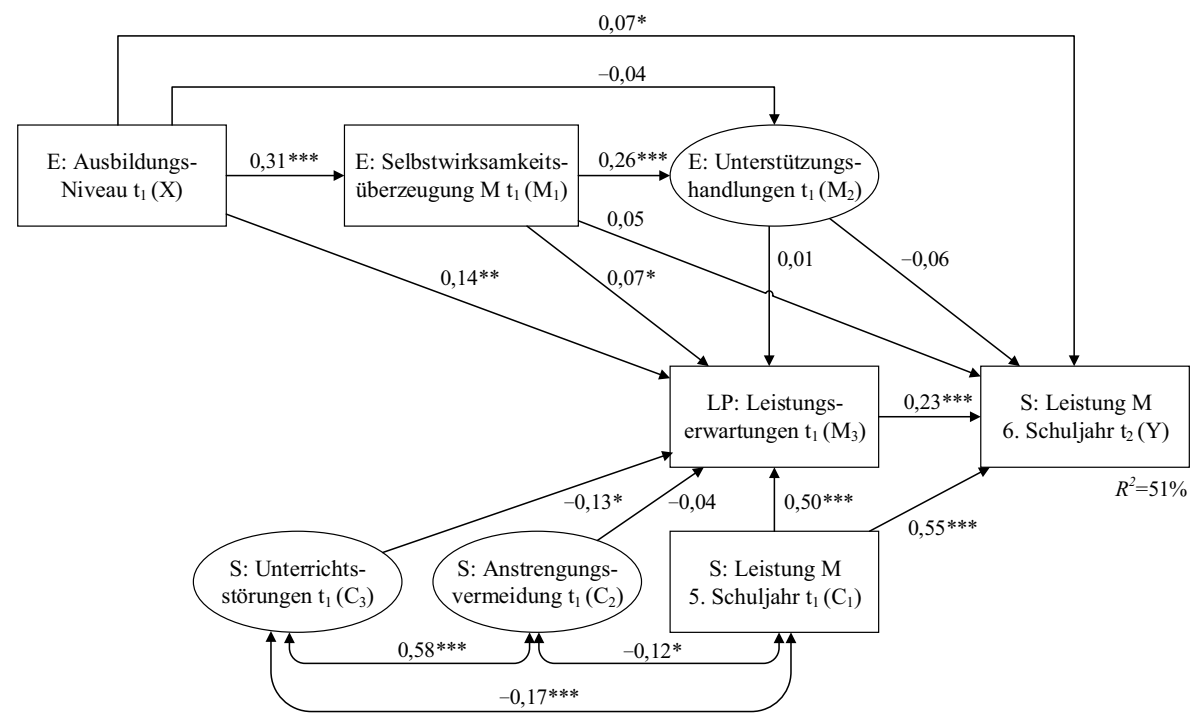

Abb. 2 Strukturgleichungsmodell zur Erklärung des Zusammenhangs zwischen dem elterlichen Ausbildungsniveau und den Leistungen von Schülerinnen und Schülern im Fach Mathematik (standardisierte $\beta$-Koeffizienten der direkten Pfade und Signifikanzen), $N=775$. ( $S$ Schülerinnen und Schüler, $E$ Eltern, $L P$ Lehrpersonen, $M$ Mathematik, $t_{1}$ erster Messzeitpunkt, $t_{2}$ zweiter Messzeitpunkt, $N$ Stichprobengröße, $X$ Unabhängige Variable, $M_{1} / M_{2} / M_{3}$ Mediatorvariablen, $Y$ Abhängige Variable, $C_{1} / C_{2} / C_{3}$ Kontrollvariablen, $R^{2}$ Varianzaufklärung. Signifikanzangaben: $\left.* * * p<0,001, * * p<0,01, * p<0,05\right)$

der indirekten Pfade $\mathrm{X} \rightarrow \mathrm{M}_{1} \rightarrow \mathrm{Y}$ und $\mathrm{X} \rightarrow \mathrm{M}_{2} \rightarrow \mathrm{Y}: \Delta B=0,01, S E=0,00, p<0,001$, $C I[0,00 ; 0,02])$. Der direkte Effekt des Ausbildungsniveaus auf die Deutschleistung im 6. Schuljahr ist nicht signifikant, weshalb davon ausgegangen werden kann, dass die drei oben genannten signifikanten Mediationen gemeinsam den direkten Effekt vollständig vermitteln.

Das Strukturgleichungsmodell in Mathematik (Abb. 2) verfügt ebenfalls über einen guten Modellfit $\left(N=775, \chi^{2}=144,17, d f=64, \mathrm{CFI}=0,96, \mathrm{RMSEA}=0,04\right.$, SRMR =0,05). Analog zum Fach Deutsch zeigt sich ein signifikanter positiver Zusammenhang zwischen dem Ausbildungsniveau der Eltern und deren Selbstwirksamkeitsüberzeugung im Fach Mathematik und kein Zusammenhang zwischen dem Ausbildungsniveau und den Unterstützungshandlungen von Eltern. Die elterliche Selbstwirksamkeitsüberzeugung in Mathematik korrespondiert positiv mit den elterlichen Unterstützungshandlungen sowie den Leistungserwartungen der Lehrpersonen. Anders als im Fach Deutsch hängt die Selbstwirksamkeitsüberzeugung in Mathematik nicht mit der Mathematikleistung im 6. Schuljahr zusammen. Die elterlichen Unterstützungshandlungen korrespondieren nicht mit der Leistung der Kinder und den Leistungserwartungen der Lehrpersonen. Die Leistungserwartungen hängen, analog zum Fach Deutsch, einerseits vom Ausbildungsniveau der Eltern ab, andererseits beeinflussen sie die Mathematikleistung im 6. Schuljahr. Analog zum Fach Deutsch ist die Kontrollvariable Leistung in Mathematik im 5. Schuljahr ein signifikanter Prädiktor für die Leistung im 6. Schuljahr sowie für die Leistungserwartungen der Lehrperson. Die Leistungserwartungen der Lehrperson hängen nicht 
mit der Anstrengungsvermeidung zusammen, jedoch negativ mit den Unterrichtsstörungen der Schülerinnen und Schüler. Die aufgeklärte Varianz der Leistungen im Fach Mathematik im 6. Schuljahr beträgt $51 \%$.

Die Ergebnisse der Mediationsanalysen (Tab. 4) zeigen, dass der Zusammenhang zwischen dem Ausbildungsniveau der Eltern und der Leistung der Schülerinnen und Schüler in Mathematik - anders als im Fach Deutsch - nicht durch die elterliche Selbstwirksamkeitsüberzeugung in Mathematik erklärt wird (H1a nicht bestätigt). Die Leistungserwartungen von Lehrpersonen erklären jedoch den Zusammenhang zwischen dem elterlichen Ausbildungsniveau und der Mathematikleistung $\left(\mathrm{X} \rightarrow \mathrm{M}_{3} \rightarrow \mathrm{Y}: \beta=0,03, S E \beta=0,01, p<0,01, C I \beta[0,01 ; 0,05], \mathrm{H} 2\right.$ bestätigt $)$. Analog zum Fach Deutsch sind auch in Mathematik diejenigen indirekten Pfade, welche die Unterstützungshandlungen der Eltern enthalten, nicht signifikant (H1b, 1c, 3b und $3 \mathrm{c}$ nicht bestätigt). Anders als im Fach Deutsch wird der Zusammenhang des elterlichen Ausbildungsniveaus und der Mathematikleistung nicht über die elterliche Selbstwirksamkeitsüberzeugung und die Leistungserwartungen der Lehrpersonen vermittelt (H3a nicht bestätigt). Weil die elterliche Selbstwirksamkeitsüberzeugung im Fach Mathematik nicht mit den Leistungen zusammenhängt, gibt es keine Unterschiede in der Vermittlung des Zusammenhangs zwischen dem Ausbildungsniveau und den Leistungen über Selbstwirksamkeitsüberzeugung und Unterstützungshandlungen der Eltern (Differenzwert der indirekten Pfade $\mathrm{X} \rightarrow \mathrm{M}_{1} \rightarrow \mathrm{Y}$ und $\mathrm{X} \rightarrow \mathrm{M}_{2} \rightarrow \mathrm{Y}$ : $\Delta B=0,01, S E=0,00, p>0,05, C I[-0,00 ; 0,01]$, H1d nicht bestätigt). Der direkte Effekt des Ausbildungsniveaus auf die Leistungen im 6. Schuljahr im Fach Mathematik bleibt signifikant (partielle Mediation).

Die Hypothese 4, welche gleiche indirekte Zusammenhänge zwischen Ausbildungsniveau und Leistungen in Deutsch und Mathematik postulierte, wird nur teilweise bestätigt. Lediglich der indirekte Effekt über die Leistungserwartungen der Lehrperson $\left(\mathrm{X} \rightarrow \mathrm{M}_{3} \rightarrow \mathrm{Y}\right)$ wird in beiden Fächern gefunden.

\section{Diskussion}

\subsection{Beantwortung der Forschungsfragen}

Herkunftsbedingte Leistungsdisparitäten wurden in vielen Studien entweder durch familiäre oder durch schulische Prozessmerkmale erklärt (Watermann und Baumert 2006; van den Bergh et al. 2010). Studien, die Merkmale der beiden Bereiche Familie und Schule in einem Modell betrachten und das gemeinsame Wirken untersuchen, sind selten (Kuperminc et al. 2008; Niederbacher und Neuenschwander 2017).

Das erste Ziel des vorliegenden Beitrags war, die sozialen Prozesse in Familie und Schule, die das Entstehen von herkunftsbedingten Leistungsparitäten bei Grundschulkindern in der Schweiz erklären, aufzuschlüsseln. Im Fach Deutsch wird der Zusammenhang zwischen dem elterlichen Ausbildungsniveau und den Leistungen von Schülerinnen und Schülern sowohl über die Elternüberzeugung (Selbstwirksamkeitsüberzeugung zur Lernunterstützung), als auch über die Lehrpersonenüberzeugung (Leistungserwartungen) vermittelt. Dies ergänzt die Ergebnisse anderer Studien (Hoover-Dempsey et al. 1992; Hollenstein et al. 2019). Zudem zeigt sich, dass die 
Eltern- und Lehrpersonenmerkmale gemeinsam Leistungsdisparitäten erklären, denn der Zusammenhang zwischen dem Ausbildungsniveau und den Deutschleistungen wird auch über beide Mediatorvariablen gemeinsam vermittelt (multiple Mediation). Dies erweitert den bestehenden Forschungsstand zur Entstehung von Leistungserwartungen von Lehrpersonen (Lorenz et al. 2016) dahingehend, dass die elterlichen Selbstwirksamkeitsüberzeugungen die Leistungserwartungen von Lehrpersonen beeinflussen. Der indirekte Zusammenhang über beide Mediatorvariablen weist zudem darauf hin, dass die vom Ausbildungsniveau geprägten Überzeugungen der Eltern die Leistungserwartungen von Lehrpersonen beeinflussen und leistungswirksam sind. Dies trägt zur Erklärung bei, wie herkunftsbedingte Chancenungerechtigkeit entsteht, was frühere Ergebnisse (Hofstetter 2017) ergänzt.

Im Fach Mathematik wird der Zusammenhang zwischen dem elterlichen Ausbildungsniveau und Leistungen lediglich über die Leistungserwartungen der Lehrperson vermittelt (analog zum Fach Deutsch). Dies bestätigt, dass Leistungserwartungen wichtige Prädiktoren der Leistungen von Schülerinnen und Schüler sind und herkunftsbedingte Leistungsdisparitäten in Mathematik erklären (Lorenz 2018; Hollenstein et al. 2019). Zwar ist der Zusammenhang zwischen der Selbstwirksamkeitsüberzeugung der Eltern in Mathematik und den Leistungserwartungen der Lehrperson, wie auch im Fach Deutsch, signifikant, die Selbstwirksamkeitsüberzeugungen spielen jedoch im Fach Mathematik bei den postulierten indirekten Zusammenhängen keine Rolle. Eccles und Harold (1993) berichten, dass die Selbstwirksamkeit von Eltern mit zunehmender Schwierigkeit der schulischen Aufgaben abnimmt. Obwohl die Eltern der vorliegenden Stichprobe im Fach Mathematik ein ähnliches Ausmaß an Selbstwirksamkeit berichten wie im Fach Deutsch, könnte es sein, dass die Selbstwirksamkeitsüberzeugungen aufgrund der zunehmenden Komplexität der Aufgaben in Mathematik ab dem 5. Grundschuljahr keine Wirkung mehr entfalten.

Insgesamt liefern diese Befunde einen Hinweis darauf, dass verschiedene Formen von Überzeugungen fachspezifisch unterschiedlich wirken. Während Leistungserwartungen in beiden Fächern signifikante Prädiktoren der Leistungen sind (Neuenschwander et al. 2007), ist dies bei den Selbstwirksamkeitsüberzeugungen nur im Fach Deutsch der Fall, nicht jedoch in Mathematik. Betrachtet man die Zusammenhänge der Konzepte in den Modellen, so finden sich zahlreiche Gemeinsamkeiten zwischen den Fächern, so beispielsweise der Zusammenhang zwischen dem Ausbildungsniveau und den Selbstwirksamkeitsüberzeugungen der Eltern im Fach Deutsch bzw. Mathematik sowie zwischen den Selbstwirksamkeitsüberzeugungen in Deutsch bzw. Mathematik und den Unterstützungshandlungen der Eltern. Der Zusammenhang zwischen Selbstwirksamkeitsüberzeugungen der Eltern in Deutsch bzw. Mathematik und den Leistungserwartungen der Lehrperson wird ebenfalls in beiden Fächern gefunden und verdeutlicht das Zusammenspiel zwischen Elternhaus und Schule.

Die Selbstwirksamkeitsüberzeugungen der Eltern sind zwar signifikante Prädiktoren für die selbstberichteten elterlichen Unterstützungshandlungen, letztere korrelieren jedoch weder mit den Leistungserwartungen der Lehrperson noch mit den Leistungen. Demnach mussten diejenigen Hypothesen, in welchen die Handlungen als Mediator fungierten, verworfen werden. Andere Studien fanden vermittelnde Effekte der Selbstwirksamkeitsüberzeugungen über Elternhandlungen auf Leistun- 
gen (Shumow und Lomax 2002; Tazouti und Jarlégan 2019), jedoch wurden andere Facetten der Elternhandlungen analysiert. Eine Erklärung für den nicht vorhandenen Effekt der Elternhandlungen könnte demnach sein, dass in der vorliegenden Studie mit der schulbezogenen Kommunikation zwischen Eltern und Kindern eine spezifische Facette der elterlichen Unterstützungshandlungen analysiert wird, welche keinen Einfluss auf die Ergebnisse standardisierter Leistungstests hat. Zukünftig sollten demnach mehrere Facetten der Elternunterstützung parallel einbezogen werden, um ein fundiertes Verständnis zur Wirkung unterschiedlicher Formen von Unterstützungshandlungen zu erhalten (Fan und Chen 2001).

Die Hypothese, dass der Zusammenhang zwischen dem Ausbildungsniveau der Eltern und Leistungen stärker über elterliche Überzeugungen wirkt als über ihre Handlungen (Jeynes 2005; Dumont et al. 2012; Neuenschwander und Niederbacher 2014), kann für das Fach Deutsch bestätigt werden. Da die hier untersuchte Facette der Unterstützungshandlungen jedoch nicht relevant ist, sollte dieser Befund nicht überinterpretiert werden.

Das zweite Ziel des Beitrags war zu überprüfen, ob sich die postulierten direkten und indirekten Zusammenhänge, welche herkunftsbedingte Leistungsdisparitäten erklären, zwischen ein- und fremd- bzw. mehrsprachigen Schülerinnen und Schülern unterscheiden und ob Mittelwertunterschiede zwischen diesen Gruppen bestehen. Die Analysen mit Bezug auf die sprachliche Herkunft von Kindern erweitern den Forschungsstand zur Entstehung von Leistungsdisparitäten, welcher bisher insbesondere Unterschiede zwischen Schülerinnen und Schülern verschiedener ethnischer Gruppen fokussierte (Davis-Kean 2005; LeFevre et al. 2010; Arens und Jude 2017). Die Ergebnisse zeigen, dass die Zusammenhänge zwischen den Variablen im Modell bei ein- und fremd- bzw. mehrsprachigen Schülerinnen und Schülern analog sind. Leistungsdisparitäten zwischen ein- und fremd- bzw. mehrsprachigen Kindern erklären sich demnach hypothesenkonform nicht durch unterschiedliche Zusammenhangsmuster, sondern weitgehend durch Unterschiede in den Ausprägungen der Variablen.

Überraschend ist das Ergebnis, dass sich einsprachige Eltern im Vergleich zu fremd- bzw. mehrsprachigen Kindern nicht signifikant in ihrem Ausbildungsniveau unterscheiden, da dieser Befund hinsichtlich des Migrationshintergrunds von Eltern in vielen Studien berichtet wird (Ramm et al. 2004; Kristen und Granato 2007). Dies verweist auf die Relevanz, Migrationshintergrund und Herkunftssprache separat zu betrachten. Zudem greift ein Erklärungsansatz, welcher auf vertikalen Unterschieden im Ausbildungsniveau basiert, möglicherweise zu kurz. Modelle der sozialen Lage (Hradil 1987; Schwenk 1999) weisen darauf hin, dass es innerhalb von „Klassen/ Schichten“ (hier „Ausbildungsniveaus“) Unterschiede zwischen Gruppen gibt, beispielsweise zwischen Ein- und Fremd- bzw. Mehrsprachigen. Selbst bei gleichem Ausbildungsniveau können demnach Fremd- oder Mehrsprachige im Vergleich zu Einsprachigen benachteiligt sein. Die Ergebnisse zu elterlichen Selbstwirksamkeitsüberzeugungen liefern ein differenziertes Bild. Fremd- bzw. mehrsprachige Eltern besitzen geringere Selbstwirksamkeitsüberzeugungen hinsichtlich der Unterstützung ihrer Kinder im Fach Deutsch. Die Unterstützung im mathematischen Bereich scheinen sie sich jedoch trotz Fremdsprachigkeit in gleichem Maße zuzutrauen. Dies bestätigt, dass die individuellen Selbstwirksamkeitsüberzeugungen bei fremd- bzw. 
mehrsprachigen Eltern nicht generell niedriger sind, sondern je nach Fach variieren (Hoover-Dempsey und Sandler 1995) und verdeutlicht den Nutzen, Selbstwirksamkeitsüberzeugungen zur Lernunterstützung fachspezifisch zu erheben. Fremdbzw. mehrsprachige Eltern berichten im Einklang mit früheren Studien (Wong und Hughes 2006) von einem höheren Ausmaß an Unterstützungshandlungen, diese sind jedoch in den vorliegenden Analysen nicht leistungsrelevant. Der Befund der geringeren Leistungserwartungen von Lehrpersonen an fremd- bzw. mehrsprachige Schülerinnen und Schüler in beiden Fächern stimmt mit den Befunden bisheriger Studien (Stahl 2007; Carigiet Reinhard 2012) überein und gibt einen Hinweis darauf, über welche Prozesse die Chancengerechtigkeit im Unterricht verletzt wird und Leistungsdisparitäten entstehen.

Die Ergebnisse zeigen, dass in denjenigen indirekten Pfaden, die herkunftsbedingte Leistungsdisparitäten erklären, in den Mediatorvariablen signifikante Mittelwertunterschiede zwischen fremd- bzw. mehrsprachigen und einsprachigen Schülerinnen und Schülern bestehen. Da die Muster der Zusammenhänge zwischen den Konzepten in den Modellen bei beiden Gruppen gleich sind (Generalisierungshypothese), haben diese Mittelwertunterschiede folgende Bedeutung: Eine geringere Selbstwirksamkeitsüberzeugung im Fach Deutsch von fremd- bzw. mehrsprachigen Eltern geht mit geringeren Leistungserwartungen der Lehrperson einher. Diese wiederum stehen in Zusammenhang mit niedrigeren Leistungen von fremd- bzw. mehrsprachigen Schülerinnen und Schülern (OECD 2016). Die Prozesse, wie herkunftsbedingte Leistungsdisparitäten entstehen, sind demnach bei ein- und fremdbzw. mehrsprachigen Schülerinnen und Schüler gleich, verlaufen aber bei Fremdbzw. Mehrsprachigen auf einem tieferen Niveau.

\subsection{Stärken und Limitationen der Studie}

Das multiperspektivisch angelegte Forschungsdesign mit Daten von Eltern, Lehrpersonen und Schülerinnen und Schülern sowie das Vorliegen von Testwerten aus standardisierten Leistungstests in zwei Fächern sind Stärken der vorliegenden Studie. Die verwendete Analysemethode der (Multigroup-)Strukturgleichungsmodelle eignete sich, um komplexe Zusammenhänge zwischen Variablen zu modellieren und die Zusammenhänge auf Gruppenunterschiede zu prüfen.

Einschränkend muss auf folgende Limitationen hingewiesen werden: Die Schülerinnen und Schüler wurden anhand der Familiensprache(n) in binäre Gruppen eingeteilt. Dies wird der Heterogenität der Gruppe der Fremd- und Mehrsprachigen nicht gerecht (Dubowy et al. 2011). Des Weiteren lässt die gewählte Operationalisierung keine Rückschlüsse auf die Sprachpraxen der Eltern zu. Um den zeitlichen Aufwand der Eltern bzw. Lehrpersonen bei der Befragung möglichst gering zu halten, wurden die elterlichen Selbstwirksamkeitsüberzeugungen in Deutsch und Mathematik sowie die Leistungserwartungen der Lehrpersonen analog zu anderen Studien (Lorenz et al. 2016; Hollenstein et al. 2019) jeweils mit Einzelitems gemessen. Dadurch sind keine Aussagen zur Validität der Konstrukte möglich. Es wurde nur eine spezifische Facette des mehrdimensionalen Konstrukts der Unterstützungshandlungen (Fan und Chen 2001) überprüft. Zudem wurde die Quantität, nicht die Qualität der Handlungen gemessen (Moroni et al. 2015). 
Fachspezifische Unterschiede konnten nur deskriptiv berichtet werden, da separate Modelle für beide Fächer berechnet wurden. Im Gegensatz zu den elterlichen Selbstwirksamkeitsüberzeugungen wurden die Leistungserwartungen der Lehrperson fächerübergreifend operationalisiert. Die Unterstützungshandlungen wurden ebenfalls nicht fachspezifisch erhoben.

Die Pfadkoeffizienten der indirekten Effekte sind mit $\beta<0,10$ in ihrer Effektstärke klein (Kline 2005), weshalb die Befunde repliziert werden sollten. Insgesamt kann jedoch angenommen werden, dass die indirekten Effekte trotz kleiner Koeffizienten in ihrer Summe nicht unerheblich sind, da Kinder über ihre gesamte Schullaufbahn diesen Prozessen ausgesetzt sind. Gleichwohl erlauben die vorliegenden Daten keine Aussagen über kausale Zusammenhänge, da die unabhängigen Variablen zeitgleich erhoben wurden. Die Reihenfolge der Variablen ist jedoch theoriebasiert (Eccles 1993; Jussim et al. 1996) begründet.

In theoretischer Hinsicht erwies es sich als gewinnbringend, die Kontexte Familie und Schule in ihrem Einfluss auf Leistungen in einem Modell zu betrachten. Die teilweise erwartungswidrigen Ergebnisse sollten nicht dazu führen, die theoretischen Modelle vorzeitig zu verwerfen, sondern zu präzisieren. Die unterschiedlichen Ergebnisse in den Fächern Deutsch und Mathematik zeigen, dass die Prozesse zur Erklärung von Leistungsdisparitäten je nach Fach verschieden sind. Zudem bestätigen die Ergebnisse einmal mehr die hohe Relevanz, Prozessmerkmale bei Analysen zu Leistungsdisparitäten miteinzubeziehen (Ehmke et al. 2006; Watermann und Baumert 2006).

\subsection{Implikationen für die Praxis}

In Bezug auf die Praxis lassen die Befunde folgende Schlussfolgerungen zu: Überzeugungen und Handlungen von Eltern und Lehrpersonen stellen einen durch Interventionen veränderbaren Bereich dar (Niklas 2015; Rubie-Davies et al. 2015). Trainingsprogramme für Eltern mit niedrigerem Ausbildungsniveau oder geringen schulsprachlichen Kompetenzen versuchen familiäre Handlungen (z. B. gemeinsames Lesen oder Hausaufgabenunterstützung; Niggli et al. 2009; Rückert et al. 2010) zu verändern. Vor dem Hintergrund, dass insbesondere elterliche Überzeugungen für Schulleistungen wirksam sind (Jeynes 2005), wäre es lohnenswert diese ebenfalls zu fördern (z. B. Leistungserwartungen, Selbstwirksamkeitsüberzeugungen).

Um einen Beitrag zu mehr Chancengerechtigkeit im Bildungssystem zu leisten, sollten Schulen in Schulentwicklungsprozessen Erwartungseffekte thematisieren. Lehrpersonen sollten dafür sensibilisiert werden, dass ihre Leistungserwartungen von familiären Merkmalen der Kinder beeinflusst sind. Trainings für Lehrpersonen zur Steigerung ihrer Leistungserwartungen (Rubie-Davies et al. 2015; Neuenschwander und Niederbacher 2019) sind vielversprechend in ihrer positiven Wirkung auf die Leistungen der Schülerinnen und Schüler. Das Aufzeigen von sozialen Prozessen, die zur Entstehung von Leistungsdisparitäten beitragen, kann Anstoß für Reflexionen in solchen Weiterbildungen sein. 
Funding Open access funding provided by FHNW University of Applied Sciences and Arts Northwestern Switzerland

Open Access Dieser Artikel wird unter der Creative Commons Namensnennung 4.0 International Lizenz veröffentlicht, welche die Nutzung, Vervielfältigung, Bearbeitung, Verbreitung und Wiedergabe in jeglichem Medium und Format erlaubt, sofern Sie den/die ursprünglichen Autor(en) und die Quelle ordnungsgemäß nennen, einen Link zur Creative Commons Lizenz beifügen und angeben, ob Änderungen vorgenommen wurden.

Die in diesem Artikel enthaltenen Bilder und sonstiges Drittmaterial unterliegen ebenfalls der genannten Creative Commons Lizenz, sofern sich aus der Abbildungslegende nichts anderes ergibt. Sofern das betreffende Material nicht unter der genannten Creative Commons Lizenz steht und die betreffende Handlung nicht nach gesetzlichen Vorschriften erlaubt ist, ist für die oben aufgeführten Weiterverwendungen des Materials die Einwilligung des jeweiligen Rechteinhabers einzuholen.

Weitere Details zur Lizenz entnehmen Sie bitte der Lizenzinformation auf http://creativecommons.org/ licenses/by/4.0/deed.de.

\section{Literatur}

Arens, A. K., \& Jude, N. (2017). Parental involvement and student achievement in two language domains: Indirect relations and generalizability across migration status. Learning and Individual Differences, $53,145-155$.

Bakker, J., Denessen, E., \& Brus-Laeven, M. (2007). Socio-economic background, parental involvement and teacher perceptions of these in relation to pupil achievement. Educational Studies, 33(2), $177-192$.

Bandura, A., Barbaranelli, C., Caprara, G. V., \& Pastorelli, C. (1996). Multifaceted impact of self-efficacy beliefs on academic functioning. Child Development, 67, 1206-1222.

Baumert, J., \& Maaz, K. (2006). Das theoretische und methodische Konzept von PISA zur Erfassung sozialer und kultureller Ressourcen der Herkunftsfamilie: Internationale und nationale Rahmenkonzeption. In J. Baumert, P. Stanat \& R. Watermann (Hrsg.), Herkunftsbedingte Disparitäten im Bildungswesen: differentielle Bildungsprozesse und Probleme der Verteilungsgerechtigkeit. Vertiefende Analysen im Rahmen von PISA 2000 (S. 11-29). Wiesbaden: VS.

van den Bergh, L., Denessen, E., Hornstra, L., Voeten, M., \& Holland, R. W. (2010). The implicit prejudiced attitudes of teachers: relations to teacher expectations and the ethnic achievement gap. American Educational Research Journal, 47(2), 497-527.

Bong, M., \& Skaalvik, E. M. (2003). Academic self-concept and self-efficacy: how different are they really? Educational psychology review, 15(1), 1-40.

Brophy, J.E. (1983). Research on the self-fulfilling prophecy and teacher expectations. Journal of Educational psychology review, 75(5), 631-661.

Carigiet Reinhard, T. (2012). Schulleistungen und Heterogenität. Eine mehrebenenanalytische Untersuchung der Bedingungsfaktoren der Schulleistungen am Ende der dritten Primarschulklasse. Bern: Haupt.

Castro, M., Expósito-Casas, E., López-Martín, E., Lizasoain, L., Navarro-Asencio, E., \& Gaviria, J.L. (2015). Parental involvement on student academic achievement: a meta-analysis. Educational Research Review, 14, 33-46.

Cheung, C. S.-S., \& Pomerantz, E.M. (2011). Parents' involvement in children's learning in the United States and China: implications for children's academic and emotional adjustment. Child Development, 82(3), 932-950.

Cheung, G.W., \& Rensvold, R.B. (2002). Evaluating goodness-of-fit indexes for testing measurement Invariance. Structural Equation Modeling: A Multidisciplinary Journal, 9(2), 233-255.

Christ, O., \& Schlüter, E. (2012). Strukturgleichungsmodelle mit Mplus. Eine praktische Einführung. München: Oldenbourg.

Chudaske, J. (2012). Sprache, Migration und schulfachliche Leistung: Einfluss sprachlicher Kompetenz auf Lese-, Rechtschreib- und Mathematikleistungen. Wiesbaden: VS.

Cohen, J. (1988). Statistical power analyses for the behavioral sciences. Hillsdale, NJ: Erlbaum: Coleman. 
Coradi Vellacott, M., \& Wolter, S.C. (2002). Soziale Herkunft und Chancengleichheit. In Bundesamt für Statistik (Hrsg.), Für das Leben gerüstet? Die Grundkompetenzen der Jugendlichen - Nationaler Bericht der Erhebung PISA 2000. Neuchâtel: Bundesamt für Statistik.

Davis-Kean, P.E. (2005). The influence of parent education and family income on child achievement: the indirect role of parental expectations and the home environment. Journal of Family Psychology, 19(2), 294-304.

Dittmann-Domenichini, N. (2014). Hören, Lesen, Schreiben - Eine Analyse schulsprachlicher Kompetenzen im Zeichen relevanter Bildungsübergänge. Zweitspracherwerb im Jugendalter, 4, 151-170.

Dittmann-Domenichini, N., Kahn, J., Rösselet, S., \& Müller, R. (2011). Sprache(n) - Schule(n) - Schulsprache(n). Ressourcen und Risikofaktoren auf dem Weg zu schulsprachlicher Kompetenz. In E. L. Wyss, S. Daniel, A. Gnach, J.-F. De Pietro \& I. De Saint-Georges (Hrsg.), Sprachkompetenzen in Ausbildung und Beruf - Übergänge und Transformationen (Bulletin VALS-ASLA, Bd. 2, Nr. 94, S. 107-128). Neuchâtel: Centre de Linguistique Appliquée.

Dubowy, M., Duzy, D., Pröscholdt, M. V., Schneider, W., Souvignier, E., \& Gold, A. (2011). Was macht den „Migrationshintergrund“ bei Vorschulkindern aus? Ein Vergleich alternativer Klassifikationskriterien und ihr Zusammenhang mit deutschen Sprachkompetenzen. Schweizerische Zeitschrift für Bildungswissenschaften, 33(3), 355-376.

Dumont, H., Trautwein, U., Lüdtke, O., Neumann, M., Niggli, A., \& Schnyder, I. (2012). Does parental homework involvement mediate the relationship between family background and educational outcomes? Contemporary Educational Psychology, 37(1), 55-69.

Dusek, J. B., \& Joseph, G. (1983). The bases of teacher expectancies. Journal of Educational Psychology, 75, 327-346.

Eccles, J.S. (1993). School and family effects on the ontogeny of children's interests, self-perceptions, and activity choice. In Nebraska symposium on motivation, 1992: Developmental perspectives on motivation (Bd. 40, S. 145-208).

Eccles, J. S., \& Harold, R. D. (1993). Parent-school involvement during the early adolescent years. Teachers College Record, 94, 568-587.

Ehmke, T., Hohensee, F., Heidemeier, H., \& Prenzel, M. (2004). Familiäre Lebensverhältnisse, Bildungsbeteiligung und Kompetenzerwerb. In M. Prenzel, J. Baumert, W. Blum, R. Lehmann, D. Leutner, M. Neubrand, R. Pekrun, G.-H. Rolff \& U. Schiefele (Hrsg.), PISA 2003. Der Bildungsstand der Jugendlichen in Deutschland - Ergebnisse des zweiten internationalen Vergleichs (S. 225-253). Münster: Waxmann.

Ehmke, T., Hohensee, F., Siegle, T., \& Prenzel, M. (2006). Soziale Herkunft, elterliche Unterstützungsprozesse und Kompetenzentwicklung. In M. Prenzel, J. Baumert, W. Blum, R. Lehmann, D. Leutner, M. Neubrand, R. Pekrun, J. Rost \& U. Schiefele (Hrsg.), PISA 2003: Untersuchungen zur Kompetenzentwicklung im Verlauf eines Schuljahres (S. 225-248). Münster: Waxmann.

Esser, H. (2006). Sprache und Integration. Die sozialen Bedingungen und Folgen des Spracherwerbs von Migranten. Frankfurt a. M.: Campus.

Fan, X., \& Chen, M. (2001). Parental involvement and students' academic achievement: a meta-analysis. Educational psychology review, 13(1), 1-22.

Graham, J. W. (2009). Missing data analysis: making it work in the real world. Annual Review of Psycho$\log y, 60,549-576$.

Gut, J., Reimann, G., \& Grob, A. (2012). Kognitive, sprachliche, mathematische und sozial-emotionale Kompetenzen als Prädiktoren späterer schulischer Leistungen: Können die Leistungen eines Kindes in den IDS dessen Schulleistungen drei Jahre später vorhersagen? Zeitschrift für Pädagogische Psychologie, 26, 213-220.

Haberman, S. J. (2009). Linking parameter estimates derived from an item response model through separate calibrations. Princeton, NJ: Educational Testing Service.

Hippmann, K., Jambor-Fahlen, S., \& Becker-Mrotzek, M. (2019). Der Einfluss familiärer Hintergrundvariablen auf die Leseleistung von Grundschulkindern im Anfangsunterricht. Zeitschrift für Erziehungswissenschaft, 22(1), 51-73.

Hofstetter, D. (2017). Die schulische Selektion als soziale Praxis. Aushandlungen von Bildungsentscheidungen beim Übergang von der Primarschule in die Sekundarstufe I. Weinheim: Beltz Juventa.

Hollenstein, L., Affolter, B., \& Brühwiler, C. (2019). Die Bedeutung der Leistungserwartung von Lehrpersonen für die Mathematikleistungen von Schülerinnen und Schülern. Zeitschrift für Erziehungswissenschaft, 22(4), 791-809.

Hoover-Dempsey, K. V., \& Sandler, H. M. (1995). Parental involvement in children's education: Why does it make a difference? Teachers College Record, 97(2), 310-331. 
Hoover-Dempsey, K. V., \& Sandler, H.M. (1997). Why do parents become involved in their children's education? Review of Educational Research, 67(1), 3-42.

Hoover-Dempsey, K. V., Bassler, O.C., \& Brissie, J.S. (1992). Explorations in parent-school relations. Journal of Educational Research, 85, 287-294.

Hoover-Dempsey, K. V., \& Sandler, H. M. (2005). The social context of parental involvement: a path to enhanced achievement. Final performance report for the OERI Grant No. R305T010673. https://ir.vanderbilt.edu/bitstream/handle/1803/7595/OERIIESfinalreport032205.pdf?sequence=1\& isAllowed=y. Zugegriffen: 18. Nov. 2019.

Hradil, S. (1987). Sozialstrukturanalyse in einer fortgeschrittenen Gesellschaft: Von Klassen und Schichten zu Lagen und Milieus. Wiesbaden: VS.

Hu, L.-T., \& Bentler, P. M. (1999). Cutoff criteria for fit indexes in covariance structure analysis: conventional criteria versus new alternatives. Structural Equation Modeling: A Multidisciplinary Journal, $6(1), 1-55$.

Hu, L. T., \& Bentler, P.M. (1998). Fit indices in covariance structure modeling: sensitivity to underparameterized model misspecification. Psychological Methods, 3, 424-453.

Jeynes, W.H. (2005). A meta-analysis of the relation of parental invovlement to urban elementary school student academic achievement. Urban Education, 40, 237-269.

Jussim, L., \& Harber, K.D. (2005). Teacher expectations and self-fulfilling prophecies: knowns and unknowns, resolved and unresolved controversies. Personality and Social Psychology Review, 9(2), $131-155$.

Jussim, L., Eccles, J.S., \& Madon, S. (1996). Social perception, social stereotypes, and teacher expectations: accuracy and the quest for the powerful self-fulfilling prophecy. In M. P. Zanna (Hrsg.), Advances in experimental social psychology (Bd. 28, S. 281-388). San Diego: Academic Press.

Jussim, L., Robustelli, S.L., \& Cain, T. R. (2009). Teacher expectations and self-fulfilling prophecies. In K. R. Wentzel \& A. Wigfield (Hrsg.), Handbook of motivation at school (S. 349-380). New York: Routledge.

Kahn, J. (2018). Mehrsprachigkeit, Sprachkompetenz und Schulerfolg. Kontexteinflüsse auf die schulsprachliche Entwicklung Ein- und Mehrsprachiger. Wiesbaden: Springer VS.

Kempert, S., Edele, A., Rauch, D., Wolf, K. M., Paetsch, J., Darsow, A., Maluch, J., \& Stanat, P. (2016). Die Rolle der Sprache für zuwanderungsbezogene Ungleichheiten im Bildungserfolg. In C. Diehl, C. Hunkler \& C. Kristen (Hrsg.), Ethnische Ungleichheiten im Bildungsverlauf (S. 157-241). Wiesbaden: Springer VS.

Kline, R. B. (2005). Principles and practice of structural equation modeling (2. Aufl.). New York: Guilford. Konsortium PISA.ch (2010). PISA 2009: Schülerinnen und Schüler der Schweiz im internationalen Vergleich. Erste Ergebnisse. Bern: BBT/EDK und Konsortium PISA.ch.

Kristen, C., \& Granato, N. (2007). The educational attainment of the second generation in Germany. Ethnicities, 7, 343-366.

Kuperminc, G., Darnell, A., \& Alvarez-Jimenez, A. (2008). Parent involvement in the academic adjustment of Latino middle and high school youth: teacher expectations and school belonging as mediators. Journal of adolescence, 31, 469-483.

Lau, R. S., \& Cheung, G. W. (2012). Estimating and comparing specific mediation effects in complex latent variable models. Organizational Research Methods, 15(1), 3-16.

LCH = Dachverband Lehrerinnen und Lehrer Schweiz (Hrsg.) (2017). Schule und Eltern: Gestaltung der Zusammenarbeit. Leitfaden für Schulen, Behörden, Elternorganisationen, Aus- und Weiterbildung. Zürich: LCH Dachverband Lehrerinnen und Lehrer Schweiz.

LeFevre, J. A., Polyzoi, E., Skwarchuk, S. L., Fast, L., \& Sowinski, C. (2010). Do home numeracy and literacy practices of Greek and Canadian parents predict the numeracy skills of kindergarten children? International Journal of Early Years Education and Urban Society, 18(1), 55-70.

Lengyel, D. (2017). Stichwort: Mehrsprachigkeitsforschung. Zeitschrift für Erziehungswissenschaft, 20(2), 153-174.

Loeys, T., Moerkerke, B., \& Vansteelandt, S. (2015). A cautionary note on the power of the test for the indirect effect in mediation analysis. Frontiers in Psychology, 5, 1549.

Lorenz, G. (2018). Selbsterfüllende Prophezeiungen in der Schule. Wiesbaden: Springer VS.

Lorenz, G., Gentrup, S., Kristen, C., Stanat, P., \& Kogan, I. (2016). Stereotype bei Lehrkräften? Eine Untersuchung systematisch verzerrter Lehrererwartungen. Kölner Zeitschrift für Soziologie und Sozialpsychologie, 68(1), 89-111.

Luplow, N., \& Smidt, W. (2019). Bedeutung von elterlicher Unterstützung im häuslichen Kontext für den Schulerfolg am Ende der Grundschule. Zeitschrift für Erziehungswissenschaft, 22(1), 153-180. 
Marsh, H. W., Hau, K. T., \& Wen, Z. (2004). In search of golden rules: comment on hypothesis-testing approaches to setting cutoff values for fit indexes and dangers in overgeneralizing Hu and Bentler's (1999) findings. Structural Equation Modeling, 11(3), 320-341.

McDonald, R.P. (1989). An index of goodness-of-fit based on noncentrality. Journal of Classification, 6(1), 97-103.

McDonald, R. P., \& Marsh, H.W. (1990). Choosing a multivariate model: Noncentrality and goodness of fit. Psychological Bulletin, 107(2), 247-255.

Midgley, C., Maehr, M. L., Hruda, L.Z., Anderman, E., Anderman, L., Freeman, K. E., Gheen, M., Kaplan, A., Kumar, R., Middleton, M. J., Nelson, J., Roeser, R., \& Urdan, T. (2000). Manual for the patterns of adaptive learning scales. Ann Arbor: University of Michigan.

Moroni, S., Dumont, H., Trautwein, U., Niggli, A., \& Baeriswyl, F. (2015). The need to distinguish between quantity and quality in research on parental involvement: the example of parental help with homework. The Journal of Educational Research, 108(5), 417-431.

Moser, U., Buff, A., Angelone, D., \& Hollenweger, J. (2011). Nach sechs Jahren Primarschule. Deutsch, Mathematik und motivational-emotionales Befinden am Ende der 6. Klasse. Zürich: Bildungsdirektion des Kantons Zürich.

Muthén, B. O., \& Satorra, A. (1995). Complex sample data in structural equation modeling. Sociological Methodology, 25, 267-316.

Muthén, L.K., \& Muthén, B.O. (2017). Mplus user's guide (8. Aufl.). Los Angeles, CA: Muthén \& Muthén. 1998-2017

Neuenschwander, M.P. (2014). Selektion beim Übergang in die Sekundarstufe I und in den Arbeitsmarkt im Vergleich. In M.P. Neuenschwander (Hrsg.), Selektion in Schule und Arbeitsmarkt: Forschungsbefunde und Praxisbeispiele (S. 63-97). Zürich: Rüegger.

Neuenschwander, M.P. (2020). Parental involvement and career decision-making: the case of Switzerland. In A. Paseka \& D. Byrne (Hrsg.), Parental involvement across European education systems (S. 135-148). London: Routledge.

Neuenschwander, M.P., \& Niederbacher, E. (2014). Elternmerkmale und Leistungsentwicklung beim Übergang in die Sekundarstufe I. Erziehung und Unterricht, 7-8, 562-568.

Neuenschwander, M.P., \& Niederbacher, E. (2019). Förderliche und gerechte Beurteilung - der SCALAAnsatz. Schweizerische Zeitschrift für Heilpädagogik, 25(7), 50-55.

Neuenschwander, M.P., Goltz, S., Balmer, T., Gasser, A., Hirt, U., Ryser, H., \& Wartenweiler, H. (2003). Eltern, Lehrpersonen und Schülerleistungen. Dokumentation des Schülerfragebogens. Bern: Kanton und Universität Bern, Stelle für Forschung und Entwicklung.

Neuenschwander, M.P., Rottermann, B., Rösselet, S., Singer, A., \& Wyler, S. (2012). Wirkungen der Selektion WiSel. Dokumentation der Lehrpersonenfragebogen der Welle 1 2011/2012 (Forschungsbericht). Solothurn: Pädagogische Hochschule der Fachhochschule Nordwestschweiz, Institut Forschung und Entwicklung.

Neuenschwander, M.P., Singer, A., Gerber, M., Rösselet, S., Rottermann, B., \& Ziehli, M. (2013). Wirkungen der Selektion WiSel. Dokumentation der Elternfragebogen der Welle 1 2011/2012 (Forschungsbericht). Solothurn: Pädagogische Hochschule der Fachhochschule Nordwestschweiz, Institut Forschung und Entwicklung.

Neuenschwander, M.P., Vida, M., Garrett, L., \& Eccles, J.S. (2007). Parents' expectations and students' achievement in two western nations. International Journal of Behavioral Development, 31(6), 594-602.

Niederbacher, E., \& Neuenschwander, M.P. (2017). Wie elterliche Selbstwirksamkeitsüberzeugungen und Lehrpersonenerwartungen die Leistungsentwicklung von Grundschulkindern mit unterschiedlicher Familiensprache erklären. Zeitschrift für Grundschulforschung, 10(2), 88-101.

Niggli, A., Wandeler, C., \& Villiger, C. (2009). Globale und bereichsspezifische Komponenten eines Elterntrainings zur Betreuung bei Lesehausaufgaben - Zusammenhänge im familiären Kontext. Unterrichtswissenschaft, 37(3), 230-245.

Niklas, F. (2015). Die familiäre Lernumwelt und ihre Bedeutung für die kindliche Kompetenzentwicklung. Psychologie in Erziehung und Unterricht, 62, 106-120.

OECD = Organisation for Economic Co-operation and Development (2016). PISA 2015 Ergebnisse (Band I): Exzellenz und Chancengerechtigkeit in der Bildung. https://www.oecd.org/berlin/themen/pisastudie/PISA_2015_Zusammenfassung.pdf. Zugegriffen: 19. Nov. 2019.

Okagaki, L., \& Frensch, P. A. (1998). Parenting and children's school achievement: a multiethnic perspective. American Educational Research Journal, 35(1), 123-144.

Ramm, G., Prenzel, M., Heidemeier, H., \& Walter, O. (2004). Soziokulturelle Herkunft: Migration. In M. Prenzel, J. Baumert, W. Blum, R. Lehmann, D. Leutner, M. Neubrand, R. Pekrun, G.-H. Rolff \& 
U. Schiefele (Hrsg.), PISA 2003. Der Bildungsstand der Jugendlichen in Deutschland - Ergebnisse des zweiten internationalen Vergleichs (S. 254-272). Münster: Waxmann.

Rubie-Davies, C. M., Peterson, E., Sibley, C. G., \& Rosenthal, R. (2015). A teacher expectation intervention: Modelling the practices of high expectation teachers. Contemporary Educational Psychology, $40,72-85$.

Rückert, E., Kunze, S., Schillert, M., \& Schulte-Körne, G. (2010). Prävention von Lese-Rechtschreibschwierigkeiten: Effekte eines Eltern-Kind-Programms zur Vorbereitung auf den Schriftspracherwerb. Kindheit und Entwicklung, 19, 82-89.

Sammons, P., Sylva, K., Melhuish, E., Siraj, I., Taggart, B., \& Hunt, S. (2008). Influences on children's attainment and progress in key stage 2: cognitive outcomes in year 6. Nottingham, United Kingdom: Departement for Children, Schools and Families.

Schneider, T., \& Pfost, M. (2013). Social and immigration-specific differences in the development of reading comprehension. A longitudinal analysis of primary school students in Germany. In M. Pfost, C. Artelt \& S. Weinert (Hrsg.), The development of reading literacy from early childhood to adolescence. Empirical findings from the Bamberg BiKS longitudinal studies (S. 151-187). Bamberg: University of Bamberg Press.

Schweizerisches Zivilgesetzbuch Artikel 302, Absatz 3. Stand: 01. Juli 2020.

Schwenk, O. G. (1999). Soziale Lagen in Deutschland. Opladen: Leske + Budrich.

Shumow, L., \& Lomax, R. (2002). Parental efficacy: predictor of parenting behavior and adolescent outcomes. Parenting, 2(2), 127-150.

SKBF = Schweizerische Koordinationsstelle für Bildungsforschung (2018). Bildungsbericht Schweiz 2018. Aarau: Schweizerische Koordinationsstelle für Bildungsforschung.

Stahl, N. (2007). Schülerwahrnehmung und -beurteilung durch Lehrkräfte. In H. Ditton (Hrsg.), Kompetenzaufbau und Laufbahnen im Schulsystem. Ergebnisse einer Längsschnittuntersuchung an Grundschulen (S. 171-198). Münster: Waxmann.

Stanat, P., Rauch, D., \& Segeritz, M. (2010). Schülerinnen und Schüler mit Migrationshintergrund. In E. Klieme, C. Artelt, J. Hartig, N. Jude, O. Köller, M. Prenzel, W. Schneider \& P. Stanat (Hrsg.), PISA 2009. Bilanz nach einem Jahrzent (S. 200-230). Münster: Waxmann.

Südkamp, A., Kaiser, J., \& Möller, J. (2012). Accuracy of teachers' judgments of students' academic achievement: a meta-analysis. Journal of Educational Psychology, 104(3), 743-762.

Tazouti, Y., \& Jarlégan, A. (2019). The mediating effects of parental self-efficacy and parental involvement on the link between family socioeconomic status and children's academic achievement. Journal of Family Studies, 25(3), 250-266.

Timmermans, A., Boer, H., \& Werf, M. (2016). An investigation of the relationship between teachers' expectations and teachers' perceptions of student attributes. Social Psychology of Education, 19, 217.

Wang, S., Rubie-Davies, C.M., \& Meissel, K. (2018). A systematic review of the teacher expectation literature over the past 30 years. Educational Research and Evaluation, 24(3-5), 124-179.

Warm, T. A. (1989). Weighted likelihood estimation of ability in item response theory. Psychometrika, 54(3), 427-450.

Watermann, R., \& Baumert, J. (2006). Entwicklung eines Strukturmodells zum Zusammenhang zwischen sozialer Herkunft und fachlichen und überfachlichen Kompetenzen: Befunde national und international vergleichender Analysen. In J. Baumert, P. Stanat \& R. Watermann (Hrsg.), Herkunftsbedingte Disparitäten im Bildungswesen. Vertiefende Analysen im Rahmen von PISA 2000 (S. 61-94). Wiesbaden: VS.

Wilder, S. (2014). Effects of parental involvement on academic achievement: a meta-synthesis. Educational Review, 66(3), 377-397.

Wong, S. W., \& Hughes, J. N. (2006). Ethnicity and language contributions to dimensions of parent involvement. School Psychology Review, 35(4), 645-662.

Yen, W. M., \& Fitzpatrick, A. R. (2006). Item response theory. In R. L. Brennan (Hrsg.), Educational measurement (S. 111-154). Westport: Praeger.

Zhao, X., Lynch Jr., J.G., \& Chen, Q. (2010). Reconsidering Baron and Kenny: myths and truths about mediation analysis. Journal of Consumer Research, 37(2), 197-206.

Zimmerman, B. J. (1995). Self-efficacy and educational development. In A. Bandura (Hrsg.), Self-efficacy in changing societies (S. 202-231). New York: Cambridge University Press. 